\title{
The model checking fingerprints of CTL operators
}

\author{
Andreas Krebs ${ }^{1}$, Arne Meier ${ }^{2}$, and Martin Mundhenk ${ }^{3}$ \\ 1 Universität Tübingen, Sand 13, 72076 Tübingen, Germany, \\ krebs@informatik. uni-tuebingen.de \\ 2 Leibniz Universität Hannover, Appelstraße 4, 30167 Hannover, Germany, \\ meier@thi. uni-hannover.de \\ 3 Friedrich-Schiller-Universität Jena, Ernst-Abbe-Platz 2, 07743 Jena, Germany, \\ martin.mundhenk@uni-jena.de
}

\begin{abstract}
The aim of this study is to understand the inherent expressive power of CTL operators. We investigate the complexity of model checking for all CTL fragments with one CTL operator and arbitrary Boolean operators. This gives us a fingerprint of each CTL operator. The comparison between the fingerprints yields a hierarchy of the operators that mirrors their strength with respect to model checking.
\end{abstract}

\section{Introduction}

Temporal logics are a long used and well-understood concept to model software specifications and computer programs by state transition semantics. The first approaches in this currently quite large area of research go back to Arthur N. Prior 23, 24]. The logics became more prominent in the 70s and 80s due to significant effort of Pnueli, Emerson, Halpern, and Clarke [8, 10,20]. Usually one distinguishes between three temporal logics: linear time logic LTL, computation tree logic CTL, and the full branching time logic CTL*. All these logics are defined as extensions of (modal) propositional logic to express properties of computer programs by introducing two path quantifiers A and E, resp., five temporal operators neXt, Until, Future, Globally, and Release. Form a syntatctic point of view, the three temporal logics differ in the way how the path quantifiers and temporal operators may be combined. The computation tree logic CTL allows operators that are combined from one path quantifier directly followed by one temporal operator. Thus there are ten different CTL operators-e.g., EX or AU.

The most important decision problems related to temporal logics are the satisfiability problem and the model checking problem. The complexity of these problems ranges between $\mathrm{P}$ and 2EXPTIME and has been classified for the general cases [7, 11, 12,22,25, 27,. Recently the satisfiability problem for all three logics has been completely classified with respect to all Boolean and temporal operator fragments 2, 16, motivated in part by the fundamental work of $\mathrm{E}$. Post 21] on Boolean functions. In the same way, the model checking problem for LTL was studied in detail [1]. The model checking problem for CTL has 


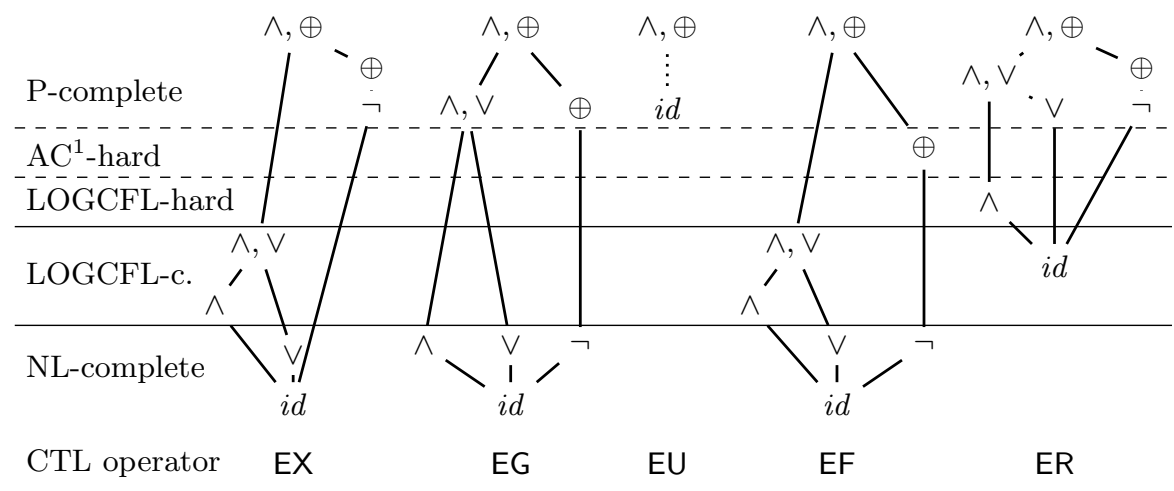

Figure 1. Overview of complexity results - the model checking fingerprints of the CTL operators.

been deeper understood in [3] who examined the complexity of CTL fragments that have arbitrary CTL operators that are combined only with all monotone Boolean operators. For model checking, there are seven relevant fragments of Boolean operators [1], but only one of these was considered in [3].

We aim to fill this gap by classifying the remaining relevant Boolean operator fragments for the computation tree logic CTL. More specifically, we examine the complexity of CTL model checking for all fragments of formulas that combine one of the ten CTL operators with one of the seven relevant fragments of Boolean operators. With our work one can completely characterize all but four of these combinations. Our classifications - informally called fingerprints - yield a preorder expressing how powerful a CTL operator is. We say a CTL operator $\mathcal{T}$ is

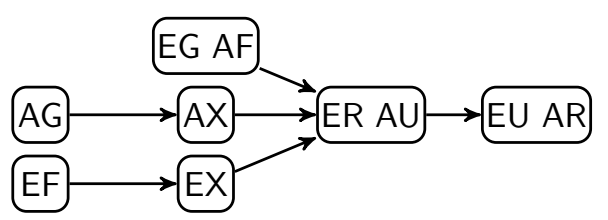

Figure 2. The mc-strength hierarchy of CTL operators that relies on their fingerprints (Fig. 11). Arrows indicate the relation $\triangleleft$. Operators $X$ and $Y$ in the same circle have the same mc-strength (i.e., $X \triangleleft Y$ and $Y \triangleleft X)$. The hierarchy is proper under the common assumptions NL $\subsetneq$ LOGCFL $\subsetneq \mathrm{P}$. mc-stronger than $\mathcal{T}^{\prime}$, in symbols $\mathcal{T}^{\prime} \triangleleft \mathcal{T}$, if for every set $B$ of Boolean operators the model checking problem for the $(\{\mathcal{T}\} \cup B)$-fragment of CTL is computationally harder than that for the $\left(\left\{\mathcal{T}^{\prime}\right\} \cup B\right)$-fragment. The resulting partial order is shown in Figure 2, It can be seen as a generalization of the notion of expressiveness of CTL fragments [13. Whereas the notion of expressiveness deals with equivalence of formulas from different fragments, our notion of mc-strength deals with equivalence of model checking instances for different fragments. Expressiveness is meaningful from a language theoretic point of view, and mc-strength from the computational complexity perspective. 
The paper is organized as follows. At first we introduce syntax and semantics of CTL, and we explain the alternating graph accessibility problems that we use in our hardness proofs (Section 2). We visit each CTL operator and show its complexity fingerprint (Sections 3.1 and 4). Finally we conclude with the resulting comparison of the mc-strength of CTL operators and an outlook to future work (Section 5). The Appendix contains the missing proofs.

\section{Preliminaries}

\subsection{Computation Tree Logic CTL}

Let PROP be a set of atomic propositions. Then the set of all well-formed CTL formulas is $\varphi::=1|p| \varphi \wedge \varphi|\varphi \vee \varphi| \varphi \oplus \varphi|\neg \varphi| \mathcal{P O} \varphi \mid \varphi \mathcal{P O}^{\prime} \varphi$, for $p \in \mathrm{PROP}, \mathcal{P} \in\{\mathrm{A}, \mathrm{E}\}, \mathcal{O} \in\{\mathrm{X}, \mathrm{F}, \mathrm{G}\}, \mathcal{O}^{\prime} \in\{\mathrm{U}, \mathrm{R}\}$. We say $\mathcal{P} \mathcal{O}$ are the unary CTL operators EX, AX, EG, AG, EF, AF and $\mathcal{P} \mathcal{O}^{\prime}$ are the binary CTL operators $\mathrm{EU}, \mathrm{AU}, \mathrm{ER}, \mathrm{AR}$. A Kripke model (for CTL) is a triple $(W, R, \xi)$, where $W$ is a finite set of states, $R: W \rightarrow W$ is a total transition relation (i.e., for all $w \in W$ there is a $w^{\prime} \in W$ with $\left.w R w^{\prime}\right)$, and $\xi: W \rightarrow 2^{\text {PROP }}$ is an assignment function.

The semantics of CTL is defined as follows on states. Let $\mathcal{M}=(W, R, \xi)$ be a Kripke model. Let $\Pi(w)$ denote the set of infinite paths starting in $w \in W$ through $(W, R)$, i.e., a path $\pi \in \Pi(w)$ is an infinite sequence $\pi=\pi[1] \pi[2] \cdots$ with $\pi[1]=w$ and $(\pi[i], \pi[i+1]) \in R$ for all $i \geq 1$.

$$
\begin{array}{ll}
\mathcal{M}, w \models 1 & \text { always, } \\
\mathcal{M}, w \models p & \text { iff } p \in \xi(w), \\
\mathcal{M}, w \models \neg \psi & \text { iff } \mathcal{M}, w \models \psi, \\
\mathcal{M}, w \models \psi \wedge \phi & \text { iff } \mathcal{M}, w \models \psi \text { and } \mathcal{M}, w \models \phi, \\
\mathcal{M}, w \models \psi \vee \phi & \text { iff } \mathcal{M}, w \models \psi \text { or } \mathcal{M}, w \models \phi, \\
\mathcal{M}, w \models \psi \oplus \phi & \text { iff }(\mathcal{M}, w \models \psi \text { and } \mathcal{M}, w \models \phi) \text { or }(\mathcal{M}, w \not \models \psi \text { and } \mathcal{M}, w \models \phi), \\
\mathcal{M}, w \models \operatorname{EX} \varphi & \text { iff } \exists \pi \in \Pi(w): \mathcal{M}, \pi[2] \models \varphi, \\
\mathcal{M}, w \models \operatorname{EF} \varphi & \text { iff } \exists \pi \in \Pi(w) \exists k \geq 1: \mathcal{M}, \pi[k] \models \varphi, \\
\mathcal{M}, w \models \operatorname{EG} \varphi & \text { iff } \exists \pi \in \Pi(w) \forall k \geq 1: \mathcal{M}, \pi[k] \models \varphi, \\
\mathcal{M}, w \models \psi \operatorname{EU} \varphi & \text { iff } \exists \pi \in \Pi(w) \exists k \geq 1: \mathcal{M}, \pi[k] \models \varphi \text { and } \forall i<k: \mathcal{M}, \pi[i] \models \psi, \\
\mathcal{M}, w \models \psi \operatorname{ER} \varphi & \text { iff } \exists \pi \in \Pi(w) \forall k \geq 1: \mathcal{M}, \pi[k] \models \varphi \text { or } \exists i<k: \mathcal{M}, \pi[i] \models \psi
\end{array}
$$

The remaining CTL operators can be expressed as duals of the above defined operators. We have the equivalences $\operatorname{AX} \varphi \equiv \neg \operatorname{EX} \neg \varphi, \operatorname{AF} \varphi \equiv \neg \mathrm{EG} \neg \varphi, \operatorname{AG} \varphi \equiv$ $\neg \mathrm{EF} \neg \varphi, \psi \mathrm{AR} \varphi \equiv \neg(\neg \psi \mathrm{EU} \neg \varphi)$, and $\psi \operatorname{AU} \varphi \equiv \neg(\neg \psi \mathrm{ER} \neg \varphi)$. Moreover, the operators EX,EG, EU are a minimal set of CTL operators that together with the Boolean operators suffice to express any from the others [13, and with the Boolean operators $\wedge, \oplus$ one can express every Boolean function. For a set $T \subseteq\{\mathrm{EX}, \mathrm{AX}, \mathrm{EG}, \mathrm{AG}, \mathrm{EF}, \mathrm{AF}, \mathrm{EU}, \mathrm{AU}, \mathrm{ER}, \mathrm{AR}, \wedge, \vee, \neg, \oplus\}$ of Boolean functions and CTL operators, a $T$-formula is a formula that has operators only from $T$. The $T$-fragment of CTL is the set of all $T$-formulas. The model checking problems for CTL fragments are defined as follows. 
Problem: CTL-MC $(T)$

Description: The model checking problem for $T$-fragments of CTL.

Input: A CTL formula $\phi$ with operators in $T \subseteq\{\mathrm{EX}, \mathrm{AX}, \mathrm{EG}$, AG, EF, AF, EU, AU, ER, AR, $\wedge, \vee, \neg, \oplus\}$, a Kripke model $\mathcal{M}=(W, R, \xi)$, and a state $w_{0} \in W$.

Question: Does $\mathcal{M}, w_{0} \models \phi$ hold?

Usually we will omit the $\{\cdot\}$ and $\cup$ in the problem notion for convenience.

Post 21 classified the lattice of all relevant sets of Boolean operators - called clones - and found a finite base for each clone. The definitions of all clones as well as the full inclusion graph can be found, for example, in [4. Whereas in general there is an infinite set of clones, for model checking luckily there are only seven different clones [1] depicted in Figure 3, where we describe the clones by their standard bases. (See, e.g., [15] for more explanations.)

\subsection{Computational Complexity}

We will make use of standard notions of complexity theory [19. In particular, we will make use of the complexity classes NL, LOGCFL, $\mathrm{AC}^{1}$, and P.

NL is the class of problems decided by nondeterministic logarithmically space bounded Turing machines. The typical complete problem is the graph accessibility problem for directed graphs REACH (given a directed graph with two nodes $s$ and $t$, is there a path form $s$ to $t$ ?). LOGCFL is the class of problems decided by nondeterministic logarithmically space bounded Turing machines, that are additionally allowed to use a stack and run in polynomial time. $\mathrm{AC}^{1}$ is the class of problems decided by alternating logarithmically space bounded Turing machines with logarithmically bounded number of alternations. We will shortly present complete problems for both of these classes. In order to prove hardness results, we will make use of logarithmic space bounded many-one reductions $\leq_{m}^{\log }$. It is known that $\mathrm{NL} \subseteq \mathrm{LOGCFL} \subseteq$

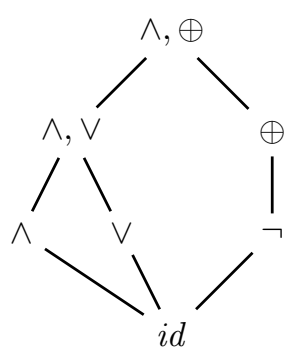

Figure 3. The Boolean clones relevant for model checking, represented by their standard bases. id denotes the clone represented without operator ("identity" of an atom). $\mathrm{AC}^{1} \subseteq \mathrm{P}$ but not whether any inclusion is strict.

Clarke, Emerson, and Sistla [7] showed that model checking for CTL is in P, and Schnoebelen [25] showed that it is P-hard.

Theorem 1 ([7, 25]). CTL-MC(EX, .., AR, $\wedge, \vee, \neg, \oplus)$ is $\mathrm{P}$-complete.

How CTL operators compare with respect to the complexity of model checking, was investigated in 3 in the following way. They completely characterize the complexity of CTL-MC $(T, \wedge, \vee)$ for every set $T$ of CTL operators. They show that this complexity is either P-complete or LOGCFL-complete. For singletons 
$S \subset\{\mathrm{AF}, \mathrm{EG}, \mathrm{AU}, \mathrm{EU}, \mathrm{AR}, \mathrm{ER}\}$ the problems $\mathrm{CTL}-\mathrm{MC}(S, \wedge, \vee)$ are P-complete, whereas for all other singletons $S \subset\{\mathrm{AX}, \mathrm{EX}, \mathrm{EF}, \mathrm{AG}\} \mathrm{CTL}-\mathrm{MC}(S, \wedge, \vee)$ is only LOGCFL-complete.

Next, we consider problems that we will use for reductions in our hardness proofs. The alternating graph accessibility problem is shown to be Pcomplete in [5]. We use the following restricted version of this problem that is very similar to Boolean circuits with and- and or-gates (and inputgates). An alternating slice graph [18] $G=(V, E)$ is a directed bipartite acyclic graph with a bipartitioning $V=V_{\exists} \cup V_{\forall}$, and a further partitioning $V=V_{0} \cup V_{1} \cup \cdots \cup V_{m}(m+1$ slices, $V_{i} \cap V_{j}=\emptyset$ if $i \neq j$ ) where

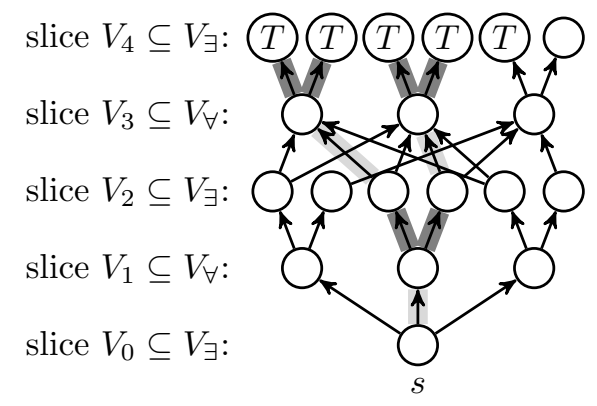

Figure 4. An instance $\langle G, s, T\rangle$ of $\operatorname{ASGAP}\left(\forall_{\text {out }}=2, \exists_{\text {in }}=1\right)$. The marked edges indicate the witness for $\operatorname{apath}_{G}(s, T)$.

$V_{\exists}=\bigcup_{i \leq m, i \text { even }} V_{i}$ and $V_{\forall}=\bigcup_{i \leq m, i \text { odd }} V_{i}$, such that $E \subseteq \bigcup_{i=0}^{m-1}\left(V_{i} \times V_{i+1}\right)$.

(All edges go from slice $V_{i}$ to slice $V_{i+1}$ for $i=0,1,2, \ldots, m-1$.) All nodes excepted those in the last slice $V_{m}$ have a positive outdegree. Nodes in $V_{\exists}$ are called existential nodes, and nodes in $V_{\forall}$ are called universal nodes. Notice that $V_{0} \subseteq V_{\exists}$ by definition. Alternating paths from node $x$ to nodes in $T \subseteq V_{m}$ are defined as follows by the property $\operatorname{apath}_{G}(x, T)$.

(1) for $x \in V_{m}$ apath $_{G}(x, T)$ iff $x \in T$

(2a) for $x \in V_{\exists}-V_{m}:$ apath $_{G}(x, T)$ iff $\exists z \in V_{\forall}:(x, z) \in E$ and $\operatorname{apath}_{G}(z, T)$

(2b) for $x \in V_{\forall}-V_{m}: \operatorname{apath}_{G}(x, T)$ iff $\forall z \in V_{\exists}:$ if $(x, z) \in E$ then $\operatorname{apath}_{G}(z, T)$

The problem ASGAP is similar to the alternating graph accessibility problem, but for the restricted class of alternating slice graphs.

Problem: ASGAP

Description: The alternating slice graph accessibility problem.

Input: $\langle G, s, T\rangle$, where $G=\left(V_{\exists} \cup V_{\forall}, E\right)$ is an alternating slice graph with slices $V_{0}, V_{1}, \ldots, V_{m}, m$ even, and $s \in V_{0}, T \subseteq$ $V_{m}$.

Question: Does $\operatorname{apath}_{G}(s, T)$ hold?

We will use also the following variant where the outdegree of $\forall$-nodes and the indegree of $\exists$-nodes is restricted. 
Problem: $\operatorname{ASGAP}\left(\forall_{\text {out }}=2, \exists_{\text {in }}=1\right)$

Description: The alternating slice graph accessibility problem with bounded degree.

Input: $\langle G, s, T\rangle$, where $G=\left(V_{\exists} \cup V_{\forall}, E\right)$ is an alternating slice graph with slices $V_{0}, V_{1}, \ldots, V_{m}$, where every node in $V_{\forall}$ has outdegree 2 and every node in $V_{\exists}-V_{0}$ has indegree 1 , and $s \in V_{0}, T \subseteq V_{m}$.

Question: Does $\operatorname{apath}_{G}(s, T)$ hold?

ASGAP $_{\log }$ is the set of all elements $\langle G, s, T\rangle$ of ASGAP, where $G$ is a graph with $n$ nodes and $m$ slices such that $m \leq \log n$. Similarly, the problem $\operatorname{ASGAP}\left(\forall_{\text {out }}=2, \exists_{\mathrm{in}}=1\right)_{\log }$ is the subset of $\operatorname{ASGAP}\left(\forall_{\text {out }}=2, \exists_{\mathrm{in}}=1\right)$ with graphs of logarithmic depth. The following completeness results are straightforward.

Theorem 2. 1. ASGAP is $\mathrm{P}$-complete [17].

2. $\operatorname{ASGAP}\left(\forall_{\text {out }}=2, \exists_{\text {in }}=1\right)$ is $\mathrm{P}$-complete.

3. ASGAP ${ }_{\log }$ is $\mathrm{AC}^{1}$-complete [18].

4. $\operatorname{ASGAP}\left(\forall_{\text {out }}=2, \exists_{\text {in }}=1\right)_{\log }$ is LOGCFL-complete.

A Kripke model $(W, R, \xi)$ contains a total graph $(W, R)$. We will use several methods to transform an alternating graph to a graph that appears as (part of) a Kripke model.

If $G=(V, E)$ is an alternating graph with slices $V_{0}, \ldots, V_{m}$, then $G^{\sharp}=$ $\left(V^{\sharp}, E^{\sharp}\right)$ is the total graph obtained from $G$ by adding a singleton slice $V_{m+1}=$ $\{e\}$ and edges from all nodes in $V_{m} \cup V_{m+1}$ to $e$. More formally, $V^{\sharp}=V \cup V_{m+1}$ and $E^{\sharp}=E \cup\left(\left(V_{m} \cup\{e\}\right) \times\{e\}\right)$.

For instances of $\operatorname{ASGAP}\left(\forall_{\text {out }}=2, \exists_{\mathrm{in}}=1\right)$, we will also apply another transformation. Let $G=(V, E)$ with slices $V_{0}, \ldots, V_{m}$ be such an instance. Every slice $V_{i} \subseteq V_{\exists}-V_{0}$ consists of nodes with indegree 1. (Remind that node(s) in $V_{0}$ have indegree 0.) Thus $V_{i} \subseteq V_{\exists}-V_{0}$ can be considered as being partitioned into sets $V_{i}^{u}:=\{v \mid(u, v) \in E\}$ for every $u \in V_{i-1}$. Then each $V_{i}^{u}$ consists of two nodes which can be assumed to be ordered arbitrarily. and we will use the notation $V_{i}^{u}=\left\{v_{u, 1}, v_{u, 2}\right\}$.

Let $\hat{V}_{i}:=\left\{\hat{v} \mid v \in V_{i}\right\}$ be a set of nodes that are "copies" of the nodes of $V_{i}$. Similarly as $V_{i}$ for even $i>0$ (i.e. $V_{i} \subseteq V_{\exists}-V_{0}$ ), $\hat{V}_{i}$ is partitioned into sets $\hat{V}_{i}^{u}=\left\{\hat{v}_{u, 1}, \hat{v}_{u, 2}\right\}$ for all $u \in V_{i-1}$. The graph $G^{b}=\left(V^{b}, E^{b}\right)$ obtained from $G$ is defined as follows. (See also Figure 5 for an example.)

$$
\begin{aligned}
V^{\mathrm{b}}:= & V \cup \bigcup_{i=0}^{m} \hat{V}_{i} \\
E^{\mathrm{b}}:= & E \cap V_{\exists} \times V_{\forall} \\
& \cup\left\{\left(u, v_{u, 1}\right) \mid u \in V_{\forall}\right\} \quad(\forall \text {-nodes have an edge to their "first" successor in } \\
& G .) \\
& \cup\left\{\left(v_{u, 1}, \hat{v}_{u, 1}\right),\left(\hat{v}_{u, 1}, v_{u, 2}\right),\left(v_{u, 2}, \hat{v}_{u, 2}\right),\left(\hat{v}_{u, 2}, \hat{v}_{u, 2}\right) \mid u \in V_{\forall}\right\} \\
& \left(\text { From each first suc. } v_{u, 1} \text { starts a path } v_{u, 1}, \hat{v}_{u, 1}, v_{u, 2}, \hat{v}_{u, 2}\right. \text { ending in a loop.) } \\
& \cup\left\{(u, \hat{u}),(\hat{u}, \hat{u}) \mid u \in V_{\forall} \cup V_{0}\right\} \\
&
\end{aligned}
$$


We will use the notion of slices also for $G^{b}$, even though there are edges between nodes in the same slice. The set of nodes $G^{b}$ is partitioned to $G^{b}=$ $V_{0}^{\mathrm{b}} \cup V_{1}^{\mathrm{b}} \cup \ldots \cup V_{m}^{\mathrm{b}}$, where slice $V_{i}^{\mathrm{b}}=V_{i} \cup \hat{V}_{i}$.

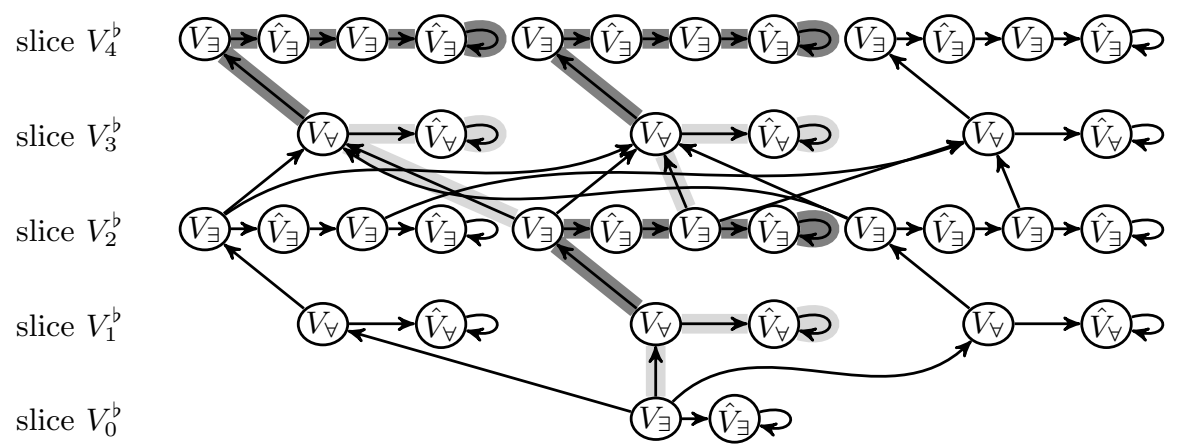

Figure 5. The graph $G^{b}$ obtained from the graph $G$ in Figure 4. The labels in the nodes indicate to which partition the node belongs. The marked edges indicate infinite paths whose collection "simulates" the witness for $\operatorname{apath}_{G}(s, T)$ in $G$.

\section{Computation Tree Logic CTL}

\subsection{Existential Until EU}

It was shown in 3 that $\mathrm{CTL}-\mathrm{MC}(\mathrm{EU}, \wedge, \vee)$ is $\mathrm{P}$-complete. We improve this result by showing that the Boolean operators are not necessary for the hardness and show that CTL-MC(EU) is P-complete (Theorem 3). Since model checking for formulas with EU as single operator reaches the maximal hardness, EU turns out to be the hardest CTL operator. We also can conclude that CTL-MC $(T)$ is P-complete for every set $T$ of Boolean functions and CTL operators that contain EU.

Technically, the proof of Theorem 3 can be seen as a guide for the P-hardness proofs for CTL-MC $(E R, \vee)$ and for CTL-MC $(E G, \oplus)$. Since the latter consider fragments with a combination of temporal and Boolean operators, their proofs are technically more involved, but the basic strategies are similar.

Theorem 3. CTL-MC(EU) is $\mathrm{P}$-complete.

Proof. The upper bound P follows from [7]. For the lower bound-P-hardnesswe give a reduction from the P-complete problem $\operatorname{ASGAP}\left(\forall_{\text {out }}=2, \exists_{\text {in }}=1\right)$. Let $\langle G, s, T\rangle$ be an instance of $\operatorname{ASGAP}\left(\forall_{\text {out }}=2, \exists_{\text {in }}=1\right)$ with $G=(V, E)$ for $V=$ $V_{\exists} \cup V_{\forall}$ with slices $V=V_{0} \cup \ldots \cup V_{m}$. Let $G^{b}=\left(V^{b}, E^{b}\right)$ be the graph obtained from $G$ as described in Section 2.2. Using $G^{b}$, we construct a Kripke model $K_{\mathrm{EU}}=\left(V^{b}, E^{b}, \xi\right)$ with assignment $\xi$ as follows (see Figure 6 for an example). 


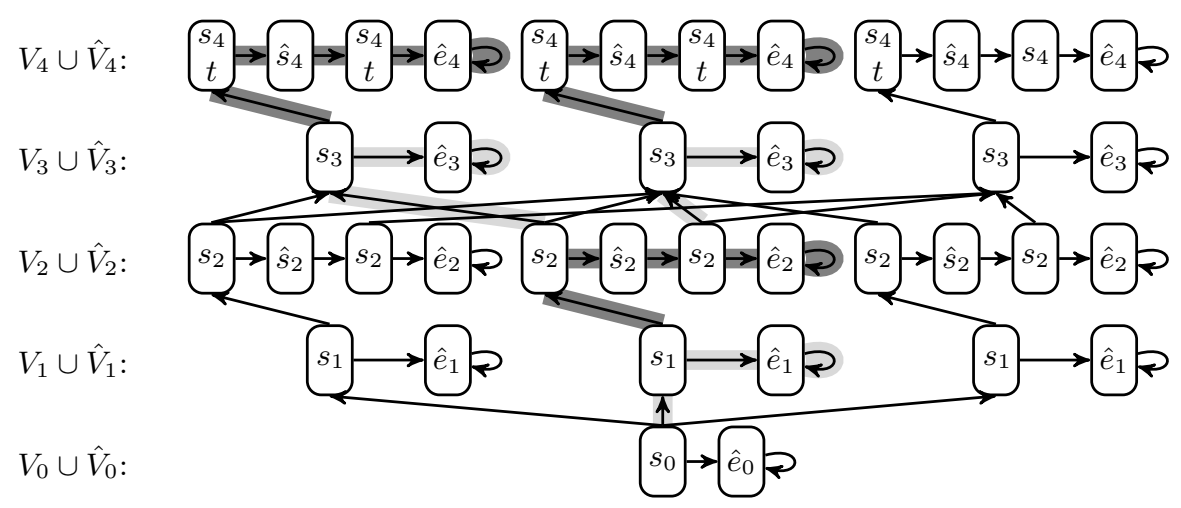

Figure 6. Example for the construction of $K_{\mathrm{EU}}$ in the proof of Theorem 3 The marked edges indicate the paths according to Claim 4.

1. $t$ is assigned to every node in $T$.

2. $s_{i}$ is assigned to every node in $V_{i_{\hat{\wedge}}}($ for $i=0,1, \ldots, m)$.

3. $\hat{s}_{i}$ is assigned to every node $v \in \hat{V}_{i}$ with $(v, v) \notin E^{\mathrm{b}}$ (for $i=0,1, \ldots, m$ ).

4. $\hat{e}_{i}$ is assigned to every node $v \in \hat{V}_{i}$ with $(v, v) \in E^{\mathrm{b}}$ (for $i=0,1, \ldots, m$ ).

The formulas $\phi_{i}$ are defined inductively for $i=m, m-1, \ldots, 0$ as follows.

$$
\phi_{i}:= \begin{cases}t, & \text { if } i=m, \\ s_{i} \operatorname{EU}\left(\left(\hat{s}_{i+1} \mathrm{EU} \phi_{i+1}\right) \mathrm{EU} \hat{e}_{i+1}\right), & \text { if } i<m .\end{cases}
$$

The Kripke model $K_{\mathrm{EU}}$ and the formulas $\phi_{i}$ are constructed in a way that simulates alternating graphs as follows. Examples for the paths used in the following Claim are indicated by marked edges in Figure 6.

Claim 4. 1. Let $w \in V_{i} \cap V_{\exists}$ for some $i<m$. Then $K_{\mathrm{EU}}, w \models \phi_{i}$ if and only if there exists a $\pi \in \Pi(w)$ with $\pi[2] \in V_{i+1}$ such that $K_{\mathrm{EU}}, \pi[2] \models \phi_{i+1}$.

2. Let $w \in V_{i} \cap V_{\forall}$ for some $i<m$. Then $K_{\mathrm{EU}}, w \models \phi_{i}$ if and only if there exists a $\pi \in \Pi(w)$ with $\pi[2], \pi[4] \in V_{i+1}$ such that $K_{\mathrm{EU}}, \pi[2] \models \phi_{i+1}$ and $K_{\mathrm{EU}}, \pi[4] \models \phi_{i+1}$.

Now we only have to use the relation between $G$ and $G^{b}$.

Claim 5. For every $i \leq m$ and every $w \in V_{i}$ holds: $K_{\mathrm{EU}}, w \models \phi_{i}$ if and only if $\operatorname{apath}_{G}(w, T)$.

The proofs of the above claims can be found in the Appendix. With Claim 5 we get that $\langle G, s, T\rangle \in \operatorname{ASGAP}\left(\forall_{\text {out }}=2, \exists_{\text {in }}=1\right)$ if and only if $K_{\mathrm{EU}}, s \models \phi_{0}$. The CTL-MC(EU) instance $\left\langle K_{\mathrm{EU}}, s, \phi_{0}\right\rangle$ can be computed in space logarithmic in the size of $G$. Thus we have shown $\operatorname{ASGAP}\left(\forall_{\text {out }}=2, \exists_{\text {in }}=1\right) \leq_{m}^{\log }$ CTL-MC(EU).

From Theorems 1 and 3 we immediately get the complete characterization of the complexity of model checking for fragments with EU-i.e., the model checking fingerprint of EU.

Theorem 6. CTL-MC(EU, $B)$ is $\mathrm{P}$-complete for every $B \subseteq\{\neg, \wedge, \vee, \oplus\}$. 


\section{The Remaining Existential Operators: Release ER, Globally EG, Next EX, and Future EF}

Let us first turn to the case of existential next EX as nothing has to be proven. Its model checking fingerprint actually is already known even though it is not always stated in the way we do it here.

Theorem 7. Let $B \subseteq\{\neg, \wedge, \vee, \oplus\}$. Then CTL-MC(EX, B) is

1. P-complete for $B \supseteq\{\neg\}$ or $B \supseteq\{\oplus\}$ [25],

2. LOGCFL-complete for $B=\{\wedge, \vee\}$ or $B=\{\wedge\}$ [3], and

3. NL-complete for $B \subseteq\{\vee\}$ (follows immediately from [15, Theorem 3.3]).

For the remainder of the results in this section we have to omit the proofs due to space constraints. However the details are all presented in the appendix. This section is structured as follows. We will state a model checking fingerprint theorem and then start to explain and discuss the results in order to give some intuition on the proof technique. Also we will mention some connections between the results, e.g., how the overall picture presents. Let us begin with the fingerprint of ER.

Theorem 8. Let $B \subseteq\{\neg, \wedge, \vee, \oplus\}$. Then $\mathrm{CTL}-\mathrm{MC}(\mathrm{ER}, B)$ is

1. P-complete for $B \supseteq\{\bigvee\}$ or $B \supseteq\{\neg\}$ or $B \supseteq\{\oplus\}$,

2. LOGCFL-hard for $B \supseteq\{\wedge\}$, and

3. LOGCFL-complete for $B=\emptyset$.

The P-completeness of CTL-MC $(\mathrm{ER}, \wedge, \vee)$ is shown in [3]. We improve this result by showing $\mathrm{P}$-hardness already for CTL-MC(ER, $\vee)$. The optimality of this hardness result is witnessed by the LOGCFL-completeness of CTL-MC(ER). Also observe that this shows that ER is not as powerful as EU. Our results are completed by the P-hardness of CTL-MC(ER, $\neg)$. Concluding, this shows that ER is strictly simpler than EU (unless LOGCFL $=\mathrm{P}$ ).

Let us now consider the case of ER with $\neg$. If $s$ is an atom that is satisfied only by a node $w$ and all its successors in a Kripke model $K$, and no successor of $w$ satisfies $s$, then $K, w \models \alpha \operatorname{ER} s$ if and only if $K, w \models \operatorname{EX} \alpha$. One can use this idea to translate the P-hardness proof of CTL-MC(EX, $\neg)$ to a P-hardness proof of CTL-MC(ER, ᄀ).

We settled complete characterizations of the complexity of CTL-MC(ER, $B)$ for fragments with ER as only CTL operator for all $B \subseteq\{\neg, \wedge, \vee, \oplus\}$ except $B=\{\wedge\}$. For CTL-MC $(\mathrm{ER}, \wedge)$ we have LOGCFL-hardness and containment in $\mathrm{P}$ (follows from [3]). A result with matching upper and lower bounds yet remains open.

Turning to the case of existentially globally operator EG, interestingly, this operator combines an existential and universal quantification in a single operator. This is worth noting as it proves itself as powerful operator from complexity point of view.

Theorem 9. Let $B \subseteq\{\neg, \wedge, \vee, \oplus\}$. Then $\mathrm{CTL}-\mathrm{MC}(\mathrm{EG}, B)$ is 
1. P-complete for $B \supseteq\{\wedge, \vee\}$ or $B \supseteq\{\oplus\}$, and

2. NL-complete for $B \subseteq\{\wedge\}$ or $B \subseteq\{\vee\}$ or $B \subseteq\{\neg\}$.

It was shown in [3] that CTL-MC(EG, $\wedge, \vee)$ is P-complete. We prove that this result is optimal by showing that CTL-MC $(E G, \wedge)$ and CTL-MC(EG, $\vee)$ are both NL-complete. Further we obtain the same characterization for CTL-MC(EG) and CTL-MC(EG, $\neg)$. The most intriguing result is the P-completeness of the fragment $\operatorname{CTL}-\mathrm{MC}(\mathrm{EG}, \oplus)$. One can reduce from $\operatorname{ASGAP}\left(\forall_{\text {out }}=2, \exists_{\mathrm{in}}=1\right)$ but it is quite demanding to explicitly argue on the chosen paths depending on the occurring exclusive-ors $\oplus$. From the model construction one can easily see similarities to the one shown in Figure 6 however one needs additional propositions labelled on the states to ensure having control on the paths.

Theorem 10. Let $B \subseteq\{\neg, \wedge, \vee, \oplus\}$. Then $\mathrm{CTL}-\mathrm{MC}(\mathrm{EF}, B)$ is

1. P-complete for $B \supseteq\{\wedge, \oplus\}$,

2. $\mathrm{AC}^{1}$-hard for $B \supseteq\{\oplus\}$, and

3. LOGCFL-complete for $\{\wedge\} \subseteq B \subseteq\{\wedge, \vee\}$, and

4. NL-complete for $B \subseteq\{\vee\}$ or $B \subseteq\{\neg\}$.

In $[3$ it is shown that CTL-MC(EF, $\wedge, \vee)$ is LOGCFL-complete. Since their hardness proof does not use $\vee$, it follows that CTL-MC(EF, $\wedge$ ) is LOGCFLcomplete, too. Moreover, CTL-MC(EF, AG, $\wedge, \vee)$ is shown to be P-complete in [3] we get P-completeness for CTL-MC $(E F, \wedge, \oplus)$. We classified almost all remaining cases. We show that the cases CTL-MC(EF), CTL-MC(EF, $\neg)$, and $\mathrm{CTL}-\mathrm{MC}(\mathrm{EF}, \mathrm{V})$ are all NL-complete. However the most interesting result is the $\mathrm{AC}^{1}$-hardness of CTL-MC $(\mathrm{EF}, \oplus)$. There are only very few problems known for which $\mathrm{AC}^{1}$ is the best shown lower bound. In fact, Cook [9] asks for natural $\mathrm{AC}^{1}$-complete problems, i.e., problems where the $\mathrm{AC}^{1}$-completeness is not forced by some logarithmic bounds in the problem definition. Chandra and Tompa 6 show an $\mathrm{AC}^{1}$-complete two-person-game that has $\mathrm{AC}^{1}$ as a straightforward upper bound and continue to ask for "less straightforward" $\mathrm{AC}^{1}$-complete problems. One such problem is the model checking problem for intuitionistic logic with one atom [18. The model checking problem for the $\{\mathrm{EF}, \oplus\}$-fragment is a very hot candidate. Anyway, it seems to be a very challenging question to show whether this problem belongs to Cook's list.

\section{Conclusion}

In this paper we aimed to present a complete complexity classification of all fragments of CTL with one CTL operator and arbitrary Boolean functions. An overview of the complexity results is given in Figure 1. We stated all our results for CTL operators that start with the existential path quantifier E. But our classification easily generalizes to the remaining CTL operators starting with the universal path quantifier A through the well-known dualities. Simply said, if CTL-MC $(T, B)$ is complete (resp. hard) for a complexity class $\mathcal{C}$, then CTL-MC(dual $(T), \operatorname{dual}(B))$ is complete (resp. hard) for the complement co- $\mathcal{C}$ of 
$\mathcal{C}$. Thus, e.g., from the $\mathrm{AC}^{1}$-hardness of CTL-MC(EF, $\oplus$ ) (Theorem 10) we immediately obtain $\mathrm{AC}^{1}$-hardness of CTL-MC $(\mathrm{AG}, \oplus)$, and from LOGCFL-completeness of CTL-MC $(E X, \wedge)$ (Theorem 7) we obtain LOGCFL-completeness of the corresponding CTL-MC $(\mathrm{AX}, \mathrm{V})$. Our results can directly be rewritten to deal not only with Boolean operators but in a more generalized view with Boolean clones as, e.g., in the work of Bauland et al. and Beyersdorff et al. 1, 3.

The only open cases for which we yet cannot prove matching upper and lower bounds are $\mathrm{CTL}-\mathrm{MC}(\mathrm{ER}, \wedge)$ and $\mathrm{CTL}-\mathrm{MC}(\mathrm{EF}, \oplus)$ and, of course, their duals CTL-MC(AU, $\vee)$ and CTL-MC(AG, $\oplus)$. Although we could not achieve an $\mathrm{AC}^{1}$ upper bound for $\mathrm{CTL}-\mathrm{MC}(\mathrm{EF}, \oplus)$, we are convinced that such a result seems closer than proving P-hardness (or some stronger hardness result than $\mathrm{AC}^{1}$ ).

Our classifications can be applied to compare the expressiveness of single CTL operators with respect to the complexity of the induced model checking problems.

Definition 11. Let $S$ and $T$ be a set of CTL operators. We say that $T$ is mcstronger than $S$ (abbreviated as $S \triangleleft T$ ), if for all sets $B$ of Boolean functions holds CTL-MC $(S, B) \leq_{m}^{\log }$ CTL-MC $(T, B)$.

The reflexive and transitive relation $\triangleleft$ for mc-strength compares what we informally called the model checking fingerprints of CTL operators. Our fingerprint theorems (Theorems 6 100) yield the hierarchy of mc-strength of CTL operators shown in Figure 2, The notion of mc-strength generalizes the notion of expressiveness [13] of CTL operators. For example, since EG $\alpha \equiv 0 \mathrm{ER} \alpha$, ER is more expressive than EG. With our notion we obtain also $E G \triangleleft E R$. But our notion yields more information about differences between several operators. For example, EX and EU have incomparable expressiveness, but we obtain EX $\triangleleft \mathrm{EU}$.

A strength-relation like $\triangleleft$ can also be defined with respect to the satisfiability problem - call it sat-strength. Whereas for model checking the set of CTL operators is partitioned into seven sets with different mc-strength (see Figure 2), from [14,16] it follows that the comparison by sat-strength yields only the following three partitions with increasing strength: $\{A F, E G\},\{E X, A X, E F, A G\}$, and $\{A U, E U, E R, A R\}$. The three notions expressiveness, sat-strength, and mcstrength intuitively compare as follows. Expressiveness relies on equivalence of formulas, sat-strength relies on equisatisfiability of formulas, and mc-strength on equisatisfaction of model checking instances.

Further work should solve the exact complexity of CTL-MC(EF, $\oplus)$, which seems to be a very challenging problem. Moreover, one should study the mcstrength of other temporal logics or of pairs of CTL operators.

\section{References}

1. M. Bauland, M. Mundhenk, T. Schneider, H. Schnoor, I. Schnoor, and H. Vollmer. The tractability of model checking for LTL: The good, the bad, and the ugly fragments. TOCL, 12(2):26, 2011.

2. M. Bauland, T. Schneider, H. Schnoor, I. Schnoor, and H. Vollmer. The complexity of generalized satisfiability for Linear Temporal Logic. LMCS, 5(1):1-21, 2009. 
3. O. Beyersdorff, A. Meier, M. Mundhenk, T. Schneider, M. Thomas, and H. Vollmer. Model checking CTL is almost always inherently sequential. LMCS, 7(2), 2011.

4. E. Böhler, N. Creignou, S. Reith, and H. Vollmer. Playing with Boolean blocks, part I: Post's lattice with applications to complexity theory. SIGACT, 34(4):38-52, 2003.

5. A. K. Chandra, D. Kozen, and L. J. Stockmeyer. Alternation. Journal of the ACM, 28:114-133, 1981.

6. A. K. Chandra and M. Tompa. The complexity of short two-person games. Discrete Applied Mathematics, 29(1):21-33, 1990.

7. E. Clarke, E. Allen Emerson, and A. Prasad Sistla. Automatic verification of finitestate concurrent systems using temporal logic specifications. TOPLAS, 8(2):244263, 1986

8. E. M. Clarke and E. Allen Emerson. Design and synthesis of synchronisation skeletons using branching time temporal logic. In Logic of Programs, volume 131 of $L N C S$, pages 52-71. Springer Verlag, 1981.

9. S. A. Cook. A taxonomy of problems with fast parallel algorithms. Information and Control, 64(1-3):2-21, 1985.

10. E. Allen Emerson and J. Y. Halpern. "Sometimes" and "not never" revisited: On branching versus linear time. Journal of the ACM, 33(1):151-178, 1986.

11. E. Allen Emerson and C. S. Jutla. The complexity of tree automata and logics of programs. SIAM Journal of Computing, 29(1):132-158, February 2000.

12. M. J. Fischer and R. E. Ladner. Propositional modal logic of programs. JCSS, 18:194-211, 1979.

13. F. Laroussinie. About the expressive power of CTL combinators. IPL, 54(6):343345, 1995.

14. A. Meier. On the Complexity of Modal Logic Variants and their Fragments. PhD thesis, Leibniz Universität Hannover, Institut für Theoretische Informatik, 2011.

15. A. Meier, J.-S. Müller, M. Mundhenk, and H. Vollmer. Complexity of model checking for logics over Kripke models. Bull. EATCS, 108:50-89, 2012.

16. A. Meier, M. Mundhenk, M. Thomas, and H. Vollmer. The complexity of satisfiability for fragments of CTL and CTL*. IJFCS, 20(05):901-918, 2009.

17. M. Mundhenk and F. Weiß. The complexity of model checking for intuitionistic logics and their modal companions. In Proc. RP'10, volume 6227 of $L N C S$, pages 146-160. Springer, 2010.

18. M. Mundhenk and F. Weiß. An $\mathrm{AC}^{1}$-complete model checking problem for intuitionistic logic. Computational Complexity, 23(4):637-669, 2014.

19. C. H. Papadimitriou. Computational Complexity. Addison-Wesley, 1994.

20. A. Pnueli. The temporal logic of programs. In Proc. 18th FOCS, pages 46-57. IEEE Computer Society Press, 1977.

21. Emil Post. The two-valued iterative systems of mathematical logic. Annals of Mathematical Studies, 5:1-122, 1941.

22. V. R. Pratt. A near-optimal method for reasoning about action. JCSS, 20(2):231254, 1980.

23. Arthur N. Prior. Time and Modality. Clarendon Press, Oxford, 1957.

24. Arthur N. Prior. Past, Present, and Future. Clarendon Press, Oxford, 1967.

25. P. Schnoebelen. The complexity of temporal logic model checking. In AiML, pages 393-436. King's College Publications, 2002.

26. M. Y. Vardi and L. Stockmeyer. Improved upper and lower bounds for modal logics of programs: Preliminary report. In STOC '85, LNCS, pages 240-251, 1985.

27. M. Y. Vardi and L. Stockmeyer. Lower bound in full (2EXPTIME-hardness for $\left.\mathrm{CTL}^{\star}-\mathrm{SAT}\right)$. Online, available at http://www.cs.rice.edu/ vardi/papers/ctl_star_lower_bound.pdf 1985. 


\section{Appendix}

\subsection{EU}

Proofs for Theorem 3; CTL-MC(EU) is $\mathrm{P}$-complete.

The basic semantical property of EU that we will use is

$K, w \models \alpha \mathrm{EU} \beta$ if and only if

(i) $K, w \models \beta$ or $(i i) K, w \models \alpha$ and $K, v \models \alpha \mathrm{EU} \beta$ for a successor $v$ of $w$.

Claim 12. For all $i<m$, all nodes $w \in V_{i}^{\mathrm{b}}$, and all $j>i$ holds: $K_{\mathrm{EU}}, w \not \models \phi_{j}$.

Proof. Let $i<m$ and $w \in V_{i}^{b}$. We proceed by induction on $j=m, m-$ $1, \ldots, i+1$. The base case is clear since $t \notin \xi(w)$ and thus $K_{\mathrm{EU}}, w \not \forall t(=$ $\left.\phi_{m}\right)$. For $j<m$, we have $K_{\mathrm{EU}}, w \not \models \phi_{j+1}$ as inductive hypothesis. Assume $K_{\mathrm{EU}}, w \models s_{j} \mathrm{EU}\left(\left(\hat{s}_{j+1} \mathrm{EU} \phi_{j+1}\right) \mathrm{EU} \hat{e}_{j+1}\right)\left(=\phi_{j}\right)$. The next steps use (1). From $s_{j} \notin \xi(w)$ follows $K_{\mathrm{EU}}, w \models\left(\hat{s}_{j+1} \mathrm{EU} \phi_{j+1}\right) \mathrm{EU} \hat{e}_{j+1}$. From $\hat{e}_{j+1} \notin \xi(w)$ then follows $K_{\mathrm{EU}}, w \models \hat{s}_{j+1} \mathrm{EU} \phi_{j+1}$, and from $\hat{s}_{j+1} \notin \xi(w)$ we conclude $K_{\mathrm{EU}}, w \models \phi_{j+1}$. This contradicts the inductive hypothesis. Thus $K_{\mathrm{EU}}, w \not \models \phi_{j}$.

The Kripke model $K_{\mathrm{EU}}$ and the formulas $\phi_{i}$ are constructed in a way that simulates alternating graphs as follows.

\section{Claim 4;}

1. Let $w \in V_{i} \cap V_{\exists}$ for some $i<m$. Then $K_{\mathrm{EU}}, w \models \phi_{i}$ if and only if there exists $a \pi \in \Pi(w)$ with $\pi[2] \in V_{i+1}$ such that $K_{\mathrm{EU}}, \pi[2] \models \phi_{i+1}$.

2. Let $w \in V_{i} \cap V_{\forall}$ for some $i<m$. Then $K_{\mathrm{EU}}, w \models \phi_{i}$ if and only if there exists a $\pi \in \Pi(w)$ with $\pi[2], \pi[4] \in V_{i+1}$ such that $K_{\mathrm{EU}}, \pi[2] \models \phi_{i+1}$ and $K_{\mathrm{EU}}, \pi[4] \models \phi_{i+1}$.

Proof of (1). For the proof direction from left to right, assume $K_{\mathrm{EU}}, w \models \phi_{i}$. Since $s_{i} \in \xi(w)$ and $s_{i} \notin \xi(v)$ for all successors $v$ of $w$, it follows from (1) that $K_{\mathrm{EU}}, v \models\left(\hat{s}_{i+1} \mathrm{EU} \phi_{i+1}\right) \mathrm{EU} \hat{e}_{i+1}$ for some successor $v$ of $w$. For $v^{\prime} \in V_{i}^{b}$ holds $\hat{s}_{i+1}, \hat{e}_{i+1} \notin \xi(v)$ and $K_{\mathrm{EU}}, v^{\prime} \not \models \phi_{i+1}$ (Claim 12), and using (11) we get $K_{\mathrm{EU}}, v^{\prime} \not \models\left(\hat{s}_{i+1} \mathrm{EU} \phi_{i+1}\right) \mathrm{EU} \hat{e}_{i+1}$. Thus there is a successor $v \in V_{i+1}$ of $w$ with $K_{\mathrm{EU}}, v \models\left(\hat{s}_{i+1} \mathrm{EU} \phi_{i+1}\right) \mathrm{EU} \hat{e}_{i+1}$. Since $\hat{s}_{i+1}, \hat{e}_{i+1} \notin \xi(v)$, this means $K_{\mathrm{EU}}, v \models$ $\phi_{i+1}$.

For the other proof direction assume $K_{\mathrm{EU}}, \pi[1] \models s_{i}$ and $K_{\mathrm{EU}}, \pi[2] \models \phi_{i+1}$ for some $\pi \in \Pi(w)$ with $\pi[2] \in V_{i+1}$. Using (1) it follows that $K_{\mathrm{EU}}, \pi[2] \models$ $\hat{s}_{i+1} \mathrm{EU} \phi_{i+1}$. Moreover, $\pi[2]$ has a successor $u \in \hat{V}_{i+1}$ with $K_{\mathrm{EU}}, u \models \hat{e}_{i+1}$. Thus from (11) follows $K_{\mathrm{EU}}, \pi[2] \models\left(\hat{s}_{i+1} \mathrm{EU} \phi_{i+1}\right) \mathrm{EU} \hat{e}_{i+1}$. Since $\pi[2]$ is a successor of $\pi[1](=w)$ and $s_{i} \in \xi(w)$, we get $K_{\mathrm{EU}}, w \models s_{i} \mathrm{EU}\left(\left(\hat{s}_{i+1} \mathrm{EU} \phi_{i+1}\right) \mathrm{EU} \hat{e}_{i+1}\right)$.

Proof of (2). This can be shown using similar arguments as above.

Claim 5; For every $i \leq m$ and every $w \in V_{i}$ holds: $K_{\mathrm{EU}}, w \models \phi_{i}$ if and only if $\operatorname{apath}_{G}(w, T)$.

Proof. The proof proceeds by induction on $i$. The base case for nodes in slice $i=m$ is straightforward. 
For the inductive step, we consider $i<m$ and $w \in V_{i}$.

First, consider $w \in V_{\exists}$. By Claim 4, we have that $K_{\mathrm{EU}}, w \models \phi_{i}$ if and only if $w$ has a successor $v \in V_{i+1}$ with $K_{\mathrm{EU}}, v \models \phi_{i+1}$. Since $v$ is also a successor of $w$ in $G$, using the inductive hypothesis, the latter is equivalent to $\operatorname{apath}_{G}(v, T)$ for a successor $v$ of $w$ in $G$. Since $w \in V_{\exists}$, this means apath $_{G}(w, T)$.

Next, consider $w \in V_{\forall}$. By Claim 4, we have that $K_{\mathrm{EU}}, w \models \phi_{i}$ if and only if there exists a path $\pi \in \Pi(w)$ with $\pi[2], \pi[4] \in V_{i+1}$ such that $K_{\mathrm{EU}}, \pi[2] \models \phi_{i+1}$ and $K_{\mathrm{EU}}, \pi[4] \models \phi_{i+1}$. Since $\pi[2]$ and $\pi[4]$ are all successors of $w$ in $G$, using the inductive hypothesis, the latter is equivalent to $\operatorname{apath}_{G}(v, T)$ for all successors $v$ of $w$ in $G$. Since $w \in V_{\forall}$, this means apath $_{G}(w, T)$.

\subsection{ER}

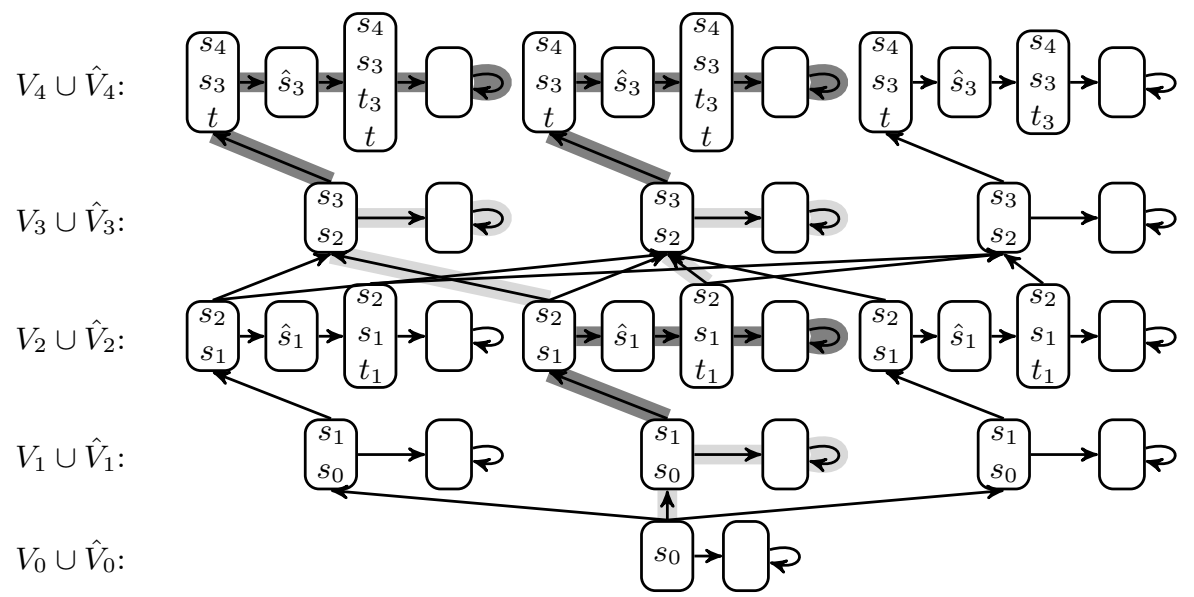

Figure 7. Kripke model $K_{(\mathrm{ER}, \mathrm{V})}$ obtained from the $\operatorname{ASGAP}\left(\forall_{\text {out }}=2, \exists_{\text {in }}=1\right)$ instance in Figure 4

Theorem 13. CTL-MC(ER, $\vee)$ is $\mathrm{P}$-complete.

Proof. Containment in $\mathrm{P}$ follows from [7. In order to show P-hardness, we logspace reduce from $\operatorname{ASGAP}\left(\forall_{\text {out }}=2, \exists_{\text {in }}=1\right)$. Let $\langle G, s, T\rangle$ be an instance of $\operatorname{ASGAP}\left(\forall_{\text {out }}=2, \exists_{\text {in }}=1\right)$, where $G=(V, E)$ and $V=V_{\exists} \cup V_{\forall}$ consists of slices $V_{0}, V_{1}, \ldots, V_{m}$ with $s \in V_{0}$ and $T \subseteq V_{m}$. Let $G^{b}=\left(V^{b}, E^{b}\right)$ be the graph obtained from $G$ as described in Section 2.2. In order to obtain the Kripke model $K_{(\mathrm{ER}, \mathrm{V})}=\left(V^{\mathrm{b}}, E^{\mathrm{b}}, \xi\right)$, it remains to define the assignment $\xi$ of atoms to sets of nodes of $G^{b}$.

1. $t$ is assigned to every node in $T$.

2. $s_{0}$ is assigned to every node in $V_{0}$. 
3. $s_{i}$ and $s_{i-1}$ are assigned to every node in $V_{i}$ for $i>0$.

4. $t_{i-1}$ is assigned to every node $v \in V_{\exists} \cap V_{i}(i>0)$ that is the second successor $v=v_{u, 2}$ of a node $u \in V_{\forall} \cap V_{i-1}$.

5. $\hat{s}_{i-1}$ is assigned to every $v \in \hat{V}_{i}(i>0)$ such that $v=\hat{v}_{u, 1}$ is the copy of the first successor of a node $u \in V_{\forall} \cap V_{i-1}$.

Nothing is assigned to nodes $\hat{v}_{u, 2}$ and to nodes in $\hat{V}_{\forall}$. Notice that all infinite paths in $G_{b}$ must eventually loop in a state $\hat{v}_{u, 2}$ that satisfies no atom at all. See Figure 7 for an example.

The formulas $\phi_{i}$ are defined inductively as follows, for $i=m, m-1, \ldots, 0$.

$$
\phi_{i}:= \begin{cases}t, & \text { if } i=m, \\ \phi_{i+1} \operatorname{ER} s_{i}, & \text { if } i<m \text { and even (slice with } \exists \text {-nodes) }, \\ t_{i} \operatorname{ER}\left(\left(\phi_{i+1} \operatorname{ER~} s_{i}\right) \vee \hat{s}_{i}\right), & \text { if } i<m \text { and odd (slice with } \forall \text {-nodes). }\end{cases}
$$

The following claim states that $\operatorname{apath}_{G}(x, T)$ corresponds to the satisfaction of formulas in the corresponding nodes in the constructed Kripke model.

Claim 14. For every $l \leq m$ and every $v \in V_{l}$ holds: $\operatorname{apath}_{G}(v, T)$ if and only if $K_{(\mathrm{ER}, \mathrm{V})}, v \models \phi_{l}$.

Proof. The proof proceeds by induction on $l$. The base case for slice $l=m$ is straightforward. For the inductive step, consider $l<m$. First consider even $l$ (slice of $\exists$-nodes), take a node $v \in V_{l} \cap V_{\exists}$, and assume

$$
K_{(\mathrm{ER}, \mathrm{V})}, v \models \phi_{l}, \quad \text { i.e. } K_{(\mathrm{ER}, \mathrm{V})}, v \models \phi_{l+1} \mathrm{ER} s_{l} .
$$

Remind that $\phi_{l+1}=t_{l+1} \operatorname{ER}\left(\left(\phi_{l+2} \operatorname{ER} s_{l+1}\right) \vee \hat{s}_{l+1}\right)$. Since $s_{l+1}, \hat{s}_{l+1} \notin \xi(v)$, it follows that $K_{(\mathrm{ER}, \mathrm{V})}, v \not \models \phi_{l+1}$. Since $s_{l} \in \xi(v)$, it follows that (2) is equivalent to

$$
\text { there exists a successor } z \text { of } v \text { with } K_{(\mathrm{ER}, \mathrm{\vee})}, z \models \phi_{l+1} \text { ER } s_{l} \text {. }
$$

The successor $\hat{v} \in \hat{V}_{l}$ of $v$ does not satisfy $s_{l}$. Thus (3) is equivalent to

$$
\text { there exists a successor } z \in V_{l+1} \text { of } v \text { with } K_{(\mathrm{ER}, \mathrm{V})}, z \models \phi_{l+1} \mathrm{ER} s_{l} \text {. }
$$

All successors $z \in V_{l+1}$ of $v$ satisfy $s_{l}$ and do not have a successor that satisfies $s_{l}$. Thus (4) is equivalent to

$$
\text { there exists a successor } z \in V_{l+1} \text { of } v \text { with } K_{(\mathrm{ER}, \mathrm{V})}, z \models \phi_{l+1} \text {. }
$$

By the inductive hypothesis and the construction of the Kripke model, this means that $v$ has a successor $z$ in $G$ with apath $_{G}(z, T)$. Since $v \in V_{\exists}$, this is equivalent to $\operatorname{apath}_{G}(v, T)$.

Now consider odd $l$ (slice of $\forall$-nodes), take a node $u \in V_{l} \cap V_{\forall}$, and assume

$$
K_{(\mathrm{ER}, \mathrm{V})}, u \models \phi_{l}, \quad \text { i.e. } K_{(\mathrm{ER}, \mathrm{V})}, u \models t_{l} \operatorname{ER}\left(\left(\phi_{l+1} \mathrm{ER} s_{l}\right) \vee \hat{s}_{l}\right) .
$$


There is no infinite path in $\Pi(u)$ that satisfies $s_{l}$ or $\hat{s}_{l}$ in every node. Thus, (6) can only be witnessed by an infinite path that passes through a node that satisfies $t_{l}$. Every such path in $\Pi(u)$ has the finite prefix $u, v_{u, 1}, \hat{v}_{u, 1}, v_{u, 2}$. Thus (6) is equivalent to

$$
\text { every node in the path } u, v_{u, 1}, \hat{v}_{u, 1}, v_{u, 2} \text { satisfies }\left(\phi_{l+1} \mathrm{ER} s_{l}\right) \vee \hat{s}_{l} \text {. }
$$

For $\hat{v}_{u, 1}$ this holds, since $\hat{s}_{l} \in \xi\left(\hat{v}_{u, 1}\right)$.

$K_{(\mathrm{ER}, \mathrm{V})}, u \models s_{l}$, but $K_{(\mathrm{ER}, \mathrm{V})}, u \not \phi_{l+1}$ because $K_{(\mathrm{ER}, \mathrm{V})}, u \forall s_{l+1}$. Thus $K_{(\mathrm{ER}, \mathrm{V})}, u \models\left(\phi_{l+1} \mathrm{ER} s_{l}\right) \vee \hat{s}_{l}$ if and only if $K_{(\mathrm{ER}, \mathrm{V})}, v_{u, 1} \models \phi_{l+1} \mathrm{ER} s_{l}$ and $K_{(\mathrm{ER}, \mathrm{V})}, v_{u, 2} \models \phi_{l+1} \mathrm{ER} s_{l}$. Since $K_{(\mathrm{ER}, \mathrm{V})}, v_{u, i} \models s_{l}$ and no successor $\hat{v}_{u, i}$ of $v_{u, i}$ satisfies $s_{l}$, it follows that $K_{(\mathrm{ER}, \mathrm{V})}, v_{u, i} \models \phi_{l+1} \mathrm{ER} s_{l}$ is equivalent to having $K_{(\mathrm{ER}, \mathrm{V})}, v_{u, i} \models \phi_{l+1}$ (for $\left.i=1,2\right)$. This yields that (7) is equivalent to

$$
K_{(\mathrm{ER}, \mathrm{V})}, v_{u, 1} \models \phi_{l+1} \text { and } K_{(\mathrm{ER}, \mathrm{V})}, v_{u, 2} \models \phi_{l+1} .
$$

By the inductive hypothesis and the construction of the Kripke model, this means that apath $_{G}(v, T)$ holds for all successors $v$ of $u$ in $G$. The latter is equivalent to apath $_{G}(u, T)$.

With Claim 14 we get that $\langle G, s, T\rangle \in \operatorname{ASGAP}\left(\forall_{\text {out }}=2, \exists_{\text {in }}=1\right)$ if and only if $K_{(\mathrm{ER}, \mathrm{V})}, s=\phi_{0}$. The CTL-MC(ER, V) instance $\left\langle K_{(\mathrm{ER}, \mathrm{V})}, s, \phi_{0}\right\rangle$ can be computed in space logarithmic in the size of $G$. Thus $\operatorname{ASGAP}\left(\forall_{\text {out }}=2, \exists_{\text {in }}=1\right) \operatorname{logspace}$ reduces to CTL-MC(ER, $\vee)$.

Theorem 15. CTL-MC(ER, ᄀ) is $\mathrm{P}$-complete.

Proof. Containment in $\mathrm{P}$ follows from [7. In order to show P-hardness, we give a reduction from ASGAP. Let $\langle G, s, T\rangle$ be an instance of ASGAP, where $G=$ $(V, E)$ for $V=V_{\exists} \cup V_{\forall}$ with slices $V_{0}, V_{1}, \ldots, V_{m}$. Let $G^{\sharp}=\left(V^{\sharp}, E^{\sharp}\right)$ be the graph obtained from $G$ as described in Section 2.2. In order to define the Kripke model $K_{(\mathrm{ER}, \neg)}=\left(V^{\sharp}, E^{\sharp}, \xi\right)$, we must give a definition of the assignment function $\xi$.

$-t$ is assigned to all nodes in $T$.

$-s_{i}$ and $s_{i-1}$ are assigned to all nodes in $V_{i}$ for $i=0,1, \ldots, m$.

The formulas $\phi_{i}$ are defined inductively for $i=m, m-1, \ldots, 0$ as follows.

$$
\phi_{i}:= \begin{cases}t, & \text { if } i=m, \\ \phi_{i+1} \text { ER } s_{i}, & \text { if } i<m \text { is even (slice of } \exists \text {-nodes), } \\ \neg\left(\neg \phi_{i+1} \text { ER } s_{i}\right), & \text { if } i<m \text { is odd (slice of } \forall \text {-nodes). }\end{cases}
$$

Notice that in the new node $e$, no atom is satisfied, and therefore no $\phi_{i}$ is satified.

Claim 16. For all $i \leq m$ and all $v \in V_{i}$ holds: $K_{(\mathrm{ER}, \neg)}, v \models \phi_{i}$ if and only if $\operatorname{apath}_{G}(v, T)$. 
The induction base $i=m$ is straightforward. For the induction step we consider $i<m$ and $v \in V_{i}$. We first consider even $i$. Since $K_{(\mathrm{ER}, \neg)}, v \not \neq s_{i+1}$ and on all paths $\pi \in \Pi(v), s_{i}$ is satisfied only in $\pi[1]=v$ and $\pi[2]$, it follows that $K_{(\mathrm{ER}, \neg)}, v \models \phi_{i+1} \mathrm{ER} s_{i}$ is equivalent to $K_{(\mathrm{ER}, \neg)}, w \models \phi_{i+1}$ for some successor $w$ of $v$. By the inductive hypothesis we obtain this to be equivalent to $\operatorname{apath}_{G}(v, T)$.

Next we consider odd $i$. Assume $K_{(\mathrm{ER}, \neg)}, v \models \neg\left(\neg \phi_{i+1}\right.$ ER $\left.s_{i}\right)$, i.e. $K_{(\mathrm{ER}, \neg)}, v \not \forall$ $\neg \phi_{i+1}$ ER $s_{i}$. Since $K_{(\mathrm{ER}, \neg)}, v \models \neg \phi_{i+1}$ and for all $\pi \in \Pi(v)$ holds that $s_{i}$ is satisfied only in $\pi[1]=v$ and $\pi[2]$, it follows that $K_{(\mathrm{ER}, \neg)}, v \not \models \neg \phi_{i+1} \mathrm{ER} s_{i}$ is equivalent to $K_{(\mathrm{ER}, \neg)}, w \forall \neg \phi_{i+1}$ for all successors $w$ of $v$. The latter means that $K_{(\mathrm{ER}, \neg)}, w \models \phi_{i+1}$ for all successors $w$ of $v$. Using the inductive hypothesis, we obtain apath $_{G}(v, T)$.

The mapping from ASGAP-instances $\langle G, s, T\rangle$ to CTL-MC(ER, $\neg)$-instances $\left\langle K_{(\mathrm{ER}, \neg)}, s, \phi_{0}\right\rangle$ can be computed in logarithmic space. With Claim 16 this yields that ASGAP logspace reduces to CTL-MC(ER, $\neg)$.

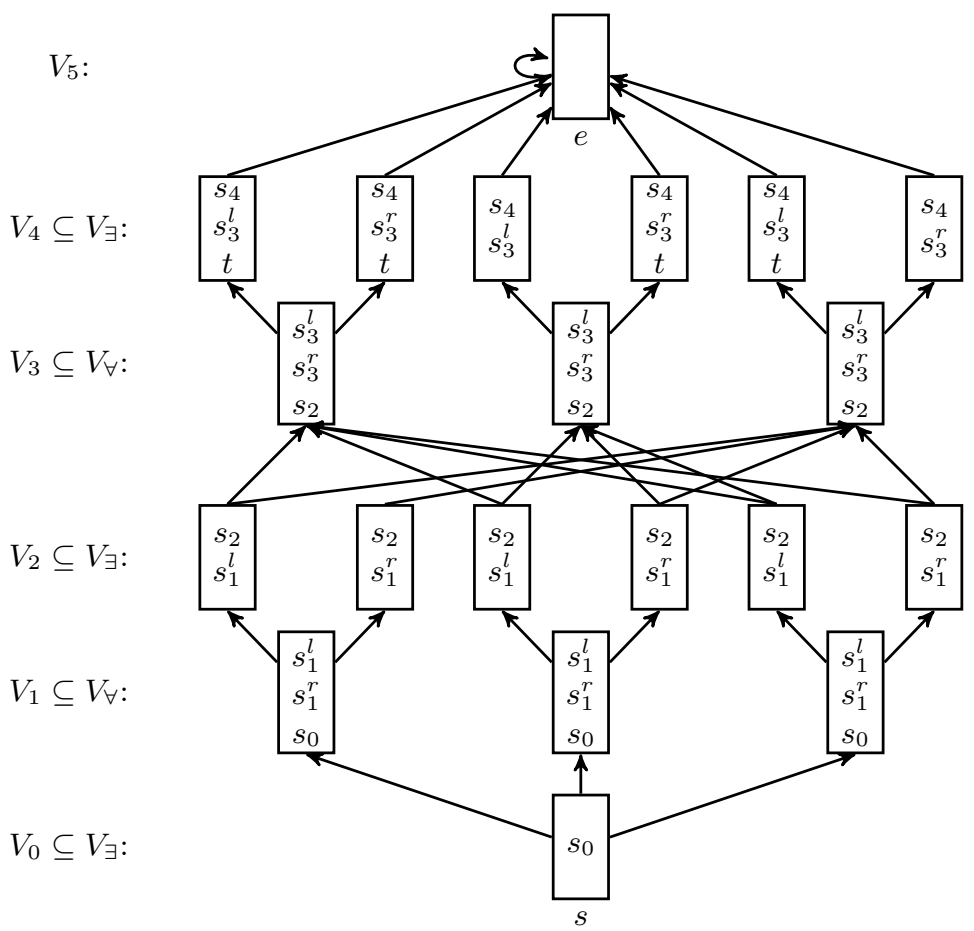

Figure 8. Kripke model $K_{\mathrm{ER}}$ obtained from the ASGAP instance in Figure 4

Theorem 17. CTL-MC(ER) is LOGCFL-hard. 


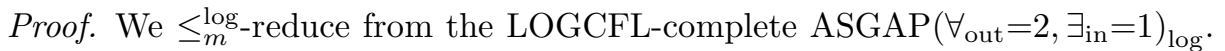
Let $\langle G, s, T\rangle$ be an instance of $\operatorname{ASGAP}\left(\forall_{\text {out }}=2, \exists_{\text {in }}=1\right)_{\log }$, where $G=(V, E)$ with $V=V_{\exists} \cup V_{\forall}$ and slices $V_{0}, \ldots, V_{m}$ for $m \leq \log |V|$. Let $G^{\sharp}=\left(V^{\sharp}, E^{\sharp}\right)$ be the graph obtained from $G$ as described in Section 2.2 In order to define the Kripke model $K_{\mathrm{ER}}=\left(V^{\sharp}, E^{\sharp}, \xi\right)$ (see Figure 8 for an example), we need to specify the assignment function $\xi$.

1. $t$ is assigned to all nodes in $T$.

2. $s_{i}$ is assigned to every node in $V_{\exists} \cap V_{i}$.

3. $s_{i-1}, s_{i}^{l}$, and $s_{i}^{r}$ are assigned to every node in $V_{\forall} \cap V_{i}$.

4. For $u \in V_{\forall} \cap V_{i}$, the two successors $v_{l}$ and $v_{r}$ of $u$ have $u$ as only predecessor. Then $s_{i}^{l}$ is assigned to $v_{l}$ and $s_{i}^{r}$ is assigned to $v_{r}$.

Notice that $V_{\exists} \cap V_{i}$ is partitioned into two sets: one to which $s_{i}^{l}$ is assigned and the other to which $s_{i}^{r}$ is assigned.

The formulas $\phi_{i}$ are defined inductively for $i=m, m-1, \ldots, 0$ as follows.

$$
\phi_{i}:= \begin{cases}t, & \text { if } i=m, \\ \phi_{i+1} \operatorname{ER} s_{i}, & \text { if } i<m \text { is even (slice of } \exists \text {-nodes), } \\ \left(\phi_{i+1} \operatorname{ER~} s_{i}^{r}\right) \operatorname{ER}\left(\phi_{i+1} \operatorname{ER} s_{i}^{l}\right), & \text { if } i<m \text { is odd (slice of } \forall \text {-nodes). }\end{cases}
$$

Claim 18. For every $i \leq m$ and every $v \in V_{i}$ holds: $K_{\mathrm{ER}}, v \models \phi_{i}$ if and only if $\operatorname{apath}_{G}(v, T)$.

The proof of the claim can be found in the Appendix. With Claim 18 we get that $\langle G, s, T\rangle \in \operatorname{ASGAP}\left(\forall_{\text {out }}=2, \exists_{\mathrm{in}}=1\right)_{\log }$ if and only if $\left\langle K_{\mathrm{ER}}, s, \phi_{0}\right\rangle \in$ CTL-MC(ER). Since the transformation can be computed in logarithmic space, it follows that $\operatorname{ASGAP}\left(\forall_{\text {out }}=2, \exists_{\mathrm{in}}=1\right)_{\log }$ logspace reduces to CTL-MC(ER).

Theorem 19. CTL-MC(ER) is LOGCFL-complete.

Proof. From Theorem 17 we have LOGCFL-hardness, hence only membership must be shown.

A right form of an $\{\mathrm{ER}\}$-formula $\psi$ is a sequence $\left\langle\alpha_{1}, \ldots, \alpha_{m}, \beta\right\rangle$ of $\{\mathrm{ER}\}$ formulas such that $\left.\psi=\alpha_{1} \operatorname{ER}\left(\alpha_{2} \operatorname{ER}\left(\alpha_{3} \operatorname{ER}\left(\cdots\left(\alpha_{m} \operatorname{ER} \beta\right)\right) \cdots\right)\right)\right)$. For example,

$$
\psi=(a \operatorname{ER} b) \operatorname{ER}((c \operatorname{ER} d) \operatorname{ER}(e \operatorname{ER} f))
$$

has, amongst others, the forms

$-\langle a \operatorname{ER} b,(c \operatorname{ER} d) \operatorname{ER}(e \operatorname{ER} f)\rangle$

$-\langle a \operatorname{ER} b, c \operatorname{ER} d, e \operatorname{ER} f\rangle$

- $\langle a \operatorname{ER} b, c \operatorname{ER} d, e, f\rangle$.

The third right form with $\beta=f$ is called atomic right form, because $f$ is an atom.

Claim 20. Let $\pi$ be a path through a Kripke model $K$. The following statements are equivalent. 
1. $\forall i \geq 1: K, \pi[i] \models \beta$

2. $\forall i \geq 1: K, \pi[i] \models\left\langle\alpha_{1}, \ldots, \alpha_{m}, \beta\right\rangle$

The proof of the Claim proceeds by induction on $m$. The base case $m=0$ is clear, because $\beta=\langle\beta\rangle$. For the inductive step $m>0$, the following holds.

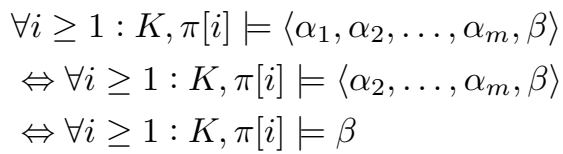

Claim 21. Let $\pi$ be a path through a Kripke model $K$, and let $k$ be an integer. The following statements are equivalent.

1. $\forall i \leq k: K, \pi[i] \models\left\langle\alpha_{1}, \ldots, \alpha_{m}, \beta\right\rangle$

2. $K, \pi[k] \models\left\langle\alpha_{1}, \ldots, \alpha_{m}, \beta\right\rangle$ and $\forall i \leq k: K, \pi[i] \models \beta$.

The proof of the Claim proceeds by induction on $m$. The base case $m=0$ is clear, because $\beta=\langle\beta\rangle$.

For the inductive step $m>0$, we consider both proof directions separately. " $\Rightarrow$ ":

$$
\begin{aligned}
& \forall i \leq k: K, \pi[i] \models\left\langle\alpha_{1}, \ldots, \alpha_{m}, \beta\right\rangle \\
& \Rightarrow \forall i \leq k \quad \exists \pi^{\prime} \in \Pi(\pi[i]) \\
& \text { (1) } \forall j \geq 1: K, \pi^{\prime}[j] \models\left\langle\alpha_{2}, \ldots, \alpha_{m}, \beta\right\rangle \text { or } \\
& \text { (2) } \exists l \geq 1: K, \pi^{\prime}[l] \models \alpha_{1} \& \forall q \leq l: K, \pi^{\prime}[q] \models\left\langle\alpha_{2}, \ldots, \alpha_{m}, \beta\right\rangle \\
& \text { (semantics of ER) } \\
& \Rightarrow \forall i \leq k \quad \exists \pi^{\prime} \in \Pi(\pi[i]) \\
& \text { (1) } \forall j \geq 1: K, \pi^{\prime}[j] \models \beta \text { or } \quad \text { (Claim) } \\
& \text { (2) } \exists l \geq 1: K, \pi^{\prime}[l] \models \alpha_{1} \& \text { (ind. hypoth.) } \\
& \forall q \leq l: K, \pi^{\prime}[q] \models \beta \& K, \pi^{\prime}[l] \models\left\langle\alpha_{2}, \ldots, \alpha_{m}, \beta\right\rangle \\
& \left.\Rightarrow \forall i \leq k: K, \pi[i] \models \beta \quad \text { (since } \pi^{\prime}[1]=\pi[i]\right) \\
& \text { "६": } \\
& K, \pi[k] \models\left\langle\alpha_{1}, \ldots, \alpha_{m}, \beta\right\rangle \text { and } \forall i \leq k: K, \pi[i] \models \beta \\
& \Rightarrow \forall i \leq k: K, \pi[i] \models \beta \text { and } \exists \pi^{\prime} \in \Pi(\pi[k]): \\
& \text { (1) } \forall j \geq 1: K, \pi^{\prime}[j] \models\left\langle\alpha_{2}, \ldots, \alpha_{m}, \beta\right\rangle \text { or } \\
& \text { (2) } \exists j \geq 1: K, \pi^{\prime}[j] \models \alpha_{1} \& \forall q \leq j: K, \pi^{\prime}[q] \models\left\langle\alpha_{2}, \ldots, \alpha_{m}, \beta\right\rangle \\
& \Rightarrow \exists \rho \in \Pi(w) \quad\left(\text { where } \rho=\pi[1] \cdots \pi[k]\left(=\pi^{\prime}[1]\right) \pi^{\prime}[2] \cdots\right) \text { : } \\
& \text { (1) } \forall j \geq 1: K, \rho[j] \models \beta \text { (using the above Claim) or } \\
& \text { (2) } \exists k^{\prime} \geq k: K, \rho\left[k^{\prime}\right] \models \alpha_{1} \& \forall i \leq k^{\prime}: K, \rho[i] \models\left\langle\alpha_{2}, \ldots, \alpha_{m}, \beta\right\rangle \text { (ind. hyp.) } \\
& \Rightarrow \forall i \leq k: K, \pi[i] \models\left\langle\alpha_{1}, \ldots, \alpha_{m}, \beta\right\rangle
\end{aligned}
$$


The atomic right form of an $\{E R\}$-formula is unique.

Claim 22. Let $\varphi$ be an $\{\mathrm{ER}\}$-formula with atomic right form $\left(\alpha_{1}, \ldots, \alpha_{m}, \beta\right)$ and $m \geq 1, K=(W, R, \xi)$ be a Kripke model, and $w \in W$. Then $K, w \models \varphi$ if and only if there exists a finite path $\pi$ through $(W, R)$ starting in $w$ with length $|\pi| \leq|W|+1$ such that

1. $K, \pi[i] \models \beta$ for all $i=1,2, \ldots,|\pi|$, and

2. (a) $|\pi|=|W|+1$, or

(b) $\quad-K, \pi[|\pi|] \models \alpha_{1}$, and

$-K, \pi[|\pi|] \models \alpha_{2} \operatorname{ER}\left(\alpha_{3} \operatorname{ER}\left(\cdots \operatorname{ER}\left(\alpha_{m} \operatorname{ER} \beta\right) \cdots\right)\right) \quad$ (i.e. the formula with atomic right form $\left.\left\langle\alpha_{2}, \ldots, \alpha_{m}, \beta\right\rangle\right)$.

$K, w \models\left\langle\alpha_{1}, \ldots, \alpha_{m}, \beta\right\rangle$ is defined as

$\exists \pi \in \Pi(w) \forall i \geq 1: K, \pi[i] \models\left\langle\alpha_{2}, \ldots, \alpha_{m}, \beta\right\rangle$ or

$\exists \pi \in \Pi(w) \exists k \geq 1: K, \pi[k] \models \alpha_{1} \& \forall j \leq k: K, \pi[j] \models\left\langle\alpha_{2}, \ldots, \alpha_{m}, \beta\right\rangle$

By Claim 20 we get that (9) is equivalent to the following.

$$
\exists \pi \in \Pi(w) \forall i \geq 1: K, \pi[i] \models \beta
$$

Since $\beta$ is an atom, (11) is equivalent to

there exists a finite path $\pi$ starting in $w$ with length $|\pi|=|W|+1$ such that $K, \pi[i] \models \beta$ for all $i=1,2, \ldots,|\pi|$.

This covers the first half (i.e. 2.a) of the claim.

Now consider (10). Using Claim 21 we get that (10) is equivalent to

$$
\exists \pi \in \Pi(w) \exists k \geq 1: \pi[k] \models \alpha_{1} \& \forall j \leq k: \pi[j] \models \beta \& \pi[k] \models\left\langle\alpha_{2}, \ldots, \alpha_{m}, \beta\right\rangle
$$

It is clear that if such a $k$ exists, then $k$ can be chosen to be $<|W|+1$. This covers the second half (i.e. 2.b) of the claim.

Algorithm 1.1 implements this algorithm according to Claim 22, It is easily seen to work in logarithmic space. The stack is used for the recursive calls. Since essentially every subformula causes one recursive call, the algorithm runs in polynomial time. Thus it is an LOGCFL algorithm.

\subsection{EG}

Theorem 23. CTL-MC $(\mathrm{EG}, \oplus)$ is $\mathrm{P}$-complete. 
Algorithm 1.1. LOGCFL machine that decides CTL-MC(ER)
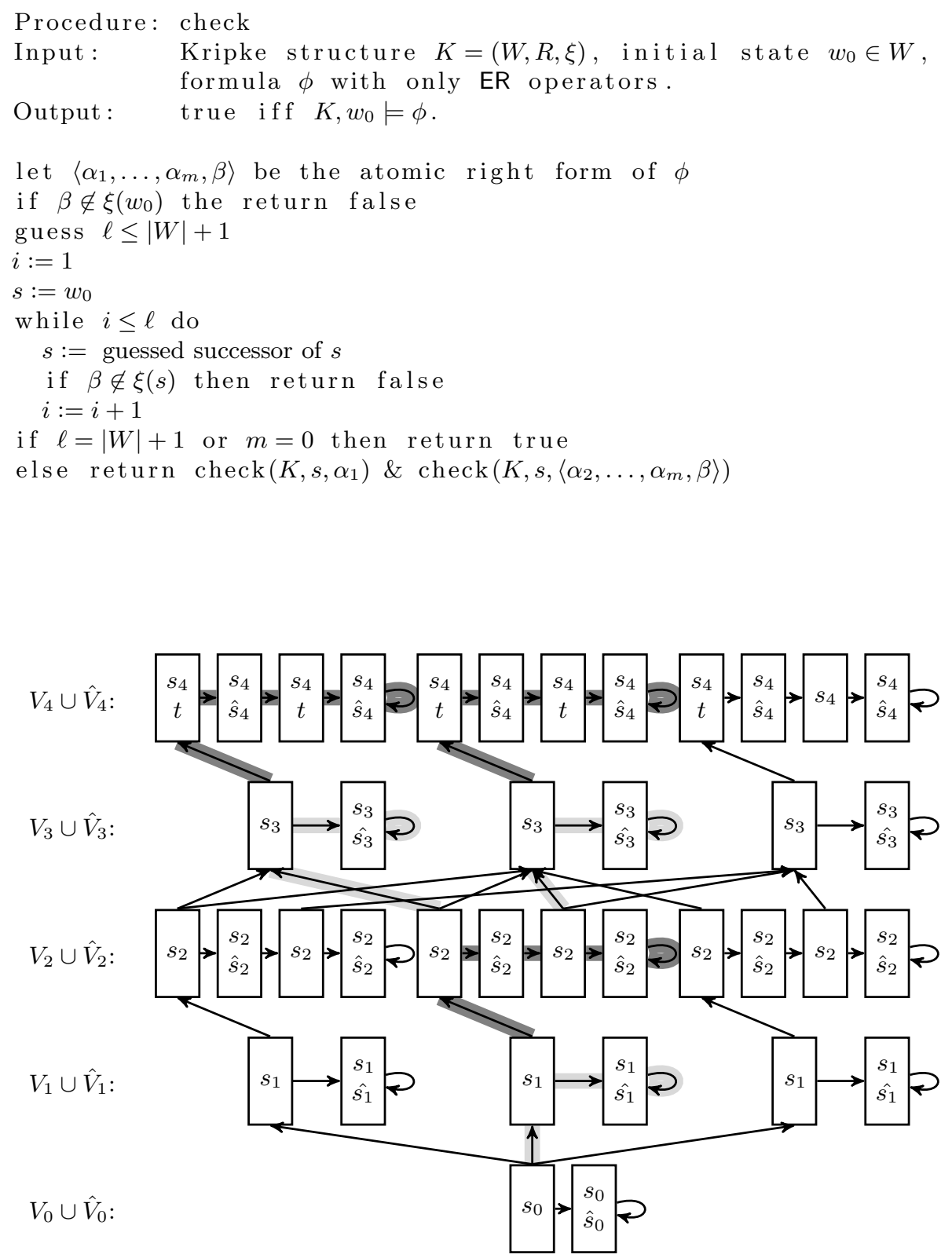

Figure 9. Kripke model $K_{(\mathrm{EG}, \oplus)}$ obtained from the $\operatorname{ASGAP}\left(\forall_{\text {out }}=2, \exists_{\mathrm{in}}=1\right)$ instance in Figure 4 
Proof. The upper bound $\mathrm{P}$ follows from [7]. For the lower bound-P-hardnesswe give a reduction from $\operatorname{ASGAP}\left(\forall_{\text {out }}=2, \exists_{\text {in }}=1\right)$. Let $\langle G, s, T\rangle$ be an instance of $\operatorname{ASGAP}\left(\forall_{\text {out }}=2, \exists_{\text {in }}=1\right)$ with $G=(V, E)$ for $V=V_{\exists} \cup V_{\forall}$ with slices $V=$ $V_{0} \cup \ldots \cup V_{m}$. Let $G^{b}=\left(V^{b}, E^{b}\right)$ be the graph obtained from $G$ as described in Section 2.2. Using $G^{b}$, we construct a Kripke model $K_{(\mathrm{EG}, \oplus)}=\left(V^{\mathrm{b}}, E^{\mathrm{b}}, \xi\right)$ with assignment $\xi$ as follows (see Figure 9 for an example).

1. $s_{i}$ is assigned to all nodes in $V_{i}^{b}$.

2. $\hat{s}_{i}$ is assigned to all nodes $\hat{V}_{i}$.

3. $t$ is assigned to all nodes in $T$.

The formulas $\varphi_{i}(i=m, m-1, \ldots, 0)$ are inductively defined as follows.

$$
\varphi_{i}= \begin{cases}t, & \text { if } i=m \\ \mathrm{EG}\left(s_{i} \oplus s_{i+2} \oplus \hat{s}_{i} \oplus \hat{s}_{i+1} \oplus \varphi_{i+1}\right), & \text { if } i<m\end{cases}
$$

We have the following easy-to-see properties of the model $K_{(\mathrm{EG}, \oplus)}$ and the formulas $\varphi_{i}$.

Claim 24. 1. For all $i \leq m$, all nodes $w \in V_{i}^{\mathrm{b}}$, and all $j>i$ holds $K_{(\mathrm{EG}, \oplus)}, w \not \models$ $\varphi_{j}$.

2. For all $i \leq m$ and all nodes $z \in \hat{V}_{i}$ holds $K_{(\mathrm{EG}, \oplus)}, z \not \neq \varphi_{i}$.

3. For all $i \leq m$ and all $u \in \hat{V}_{i}$ with $(u, u) \in E^{b}$ holds $K_{(\mathrm{EG}, \oplus)}, u \models \varphi_{i-1}$.

We sketch the proof. For [1 $K_{(\mathrm{EG}, \oplus)}, w \not \varphi_{j}$ since no atoms that appear in $\varphi_{j}$ are assigned to node $w$ in slice $i<j$.

For 22 By the definition of $\xi$ we have $z \in \xi\left(s_{i}\right)$ and $z \in \xi\left(\hat{s}_{i}\right)$, and $z \notin \xi\left(s_{i+2}\right)$ and $z \notin \xi\left(\hat{s}_{i+1}\right)$. With case 1 we also have $K_{(\mathrm{EG}, \oplus)}, z \not \models \varphi_{i+1}$. Thus $K_{(\mathrm{EG}, \oplus)}, z \not \models$ $s_{i} \oplus s_{i+2} \oplus \hat{s}_{i} \oplus \hat{s}_{i+1} \oplus \varphi_{i+1}$, and consequently $K_{(\mathrm{EG}, \oplus)}, z \not \forall \varphi_{i}$.

For [3] For $\varphi_{i-1}=\mathrm{EG}\left(s_{i-1} \oplus s_{i+1} \oplus \hat{s}_{i-1} \oplus \hat{s}_{i} \oplus \varphi_{i}\right)$, we have that $\hat{s}_{i} \in \xi(u)$ and $s_{i-1}, s_{i+1}, \hat{s}_{i-1} \notin \xi(u)$. From 2 we get $K_{(\mathrm{EG}, \oplus)}, u \not \models \varphi_{i}$. Thus, $K_{(\mathrm{EG}, \oplus)}, u \models$ $s_{i-1} \oplus s_{i+1} \oplus \hat{s}_{i-1} \oplus \hat{s}_{i} \oplus \varphi_{i}$. Since all infinite paths $\pi \in \Pi(u)$ only loop through $u$ (e.g. $\pi[k]=u$ for all $k \geq 1$ ), it follows that $K_{(\mathrm{EG}, \oplus)}, u \models \varphi_{i-1}$.

Claim 25. For all $i \leq m$, all nodes $w \in V_{i}^{\mathrm{b}}$, and all $j \leq i-2$ holds: $K_{(\mathrm{EG}, \oplus)}, w \not \models$ $\varphi_{j}$.

The proof is by induction on $i$.

- Base case $i=m$. Consider $w \in V_{m}^{\mathrm{b}}$. Notice that every infinite path $\pi \in \Pi(w)$ eventually loops in a node $u_{w} \in V_{m}$ with $\left(u_{w}, u_{w}\right) \in E^{\mathrm{b}}$. Since $K, v \models \mathrm{EG} \alpha$ if and only if $K, v \models \alpha$ and $K, v^{\prime} \models \mathrm{EG} \alpha$ for some successor $v^{\prime}$ of $v$, it suffices to show that $K_{(\mathrm{EG}, \oplus)}, u_{w} \not \neq \varphi_{j}$. We proceed by induction on $j$, where $j=m-2$ is the base case. Since $K_{(\mathrm{EG}, \oplus)}, u_{w} \models \varphi_{m-1}$ (Claim 24](3)), it follows that $K_{(\mathrm{EG}, \oplus)}, u_{w} \not s_{m-2} \oplus s_{m} \oplus \hat{s}_{m-2} \oplus \hat{s}_{m-1} \oplus \varphi_{m-1}$, and thus $K_{(\mathrm{EG}, \oplus)}, u_{w} \not \models \varphi_{m-2}$. (Generally, a formula $\mathrm{EG} \alpha$ is satisfied in a node $u$ if and only if $u \models \alpha$ and $v \models \mathrm{EG} \alpha$ for some successor $v$ of $u$.)

For $j<m-2$, we have the inductive hypothesis $K_{(\mathrm{EG}, \oplus)}, u_{w} \forall \varphi_{j+1}$. Since $K_{(\mathrm{EG}, \oplus)}, u_{w} \not \models s_{j} \oplus s_{j+2} \oplus \hat{s}_{j} \oplus \hat{s}_{j+1}$, it follows that $K_{(\mathrm{EG}, \oplus)}, u_{w} \not \models s_{j} \oplus s_{j+2} \oplus$ $\hat{s}_{j} \oplus \hat{s}_{j+1} \oplus \varphi_{j+1}$ and thus $K_{(\mathrm{EG}, \oplus)}, u_{w} \not \models \varphi_{j}$. 
- Inductive step $i<m$. Consider node $w \in V_{i}^{b}$. Again we proceed by induction on $j$.

- Base case $j=i-2$ for $\varphi_{j}=\mathrm{EG}\left(s_{j} \oplus s_{j+2} \oplus \hat{s}_{j} \oplus \hat{s}_{j+1} \oplus \varphi_{j+1}\right)$. Since no states in slices $\geq i$ satisfy $s_{j}, \hat{s}_{j}$, and $\hat{s}_{j+1}$, and $K_{(\mathrm{EG}, \oplus)}, w \models s_{j+2}\left(=s_{i}\right)$, it follows that $K_{(\mathrm{EG}, \oplus)}, w \models \varphi_{j}$ iff $K_{(\mathrm{EG}, \oplus)}, w \models \mathrm{EG}\left(s_{j+2} \oplus \varphi_{j+1}\right)$. By inductive hypothesis we have $K_{(\mathrm{EG}, \oplus)}, v \not=\varphi_{j+1}$ for all $v \in V_{i+1}^{b}$. These nodes $v$ do not satisfy $s_{j+2}$. Therefore $K_{(\mathrm{EG}, \oplus)}, w \models \mathrm{EG}\left(s_{j+2} \oplus \varphi_{j+1}\right)$ only holds, if it is witnessed by a path that stays in slice $V_{i}^{b}$. This path eventually loops in a node $u_{w} \in V_{i}$ with $\left(u_{w}, u_{w}\right) \in E^{b}$. By Claim 24(3) we know $K_{(\mathrm{EG}, \oplus)}, u_{w} \models \varphi_{j+1}$ (since $j+1=i-1$ ). Consider $\varphi_{j}=$ $\mathrm{EG}\left(s_{j} \oplus s_{j+2} \oplus \hat{s}_{j} \oplus \hat{s}_{j+1} \oplus \varphi_{j+1}\right)$. We have that $s_{j+2}$ and $\varphi_{j+1}$ are the only "parts" of $\varphi_{j}$ that are satisfied in $u_{w}$. Thus $K_{(\mathrm{EG}, \oplus)}, u_{w} \not$ $s_{j} \oplus s_{j+2} \oplus \hat{s}_{j} \oplus \hat{s}_{j+1} \oplus \varphi_{j+1}$, and therefore $K_{(\mathrm{EG}, \oplus)}, u_{w} \not \models \varphi_{j}$.

Since every path from $w$ that stays in slice $i$ ends in such a node $u_{w}$, we get that $K_{(\mathrm{EG}, \oplus)}, w \not \models \varphi_{j}$.

- Inductive step $j<i-2$. Consider $\varphi_{j}=\mathrm{EG}\left(s_{j} \oplus s_{j+2} \oplus \hat{s}_{j} \oplus \hat{s}_{j+1} \oplus\right.$ $\left.\varphi_{j+1}\right)$. By inductive hypothesis we know $K_{(\mathrm{EG}, \oplus)}, w \not \models \varphi_{j+1}$. Moreover, $s_{j}, s_{j+2}, \hat{s}_{j}, \hat{s}_{j+1} \notin \xi(w)$. Therefore $K_{(\mathrm{EG}, \oplus)}, w \not \models \varphi_{j}$.

Claim 26. For every $i \leq m$ and every $w \in V_{i}$ holds: $K_{(\mathrm{EG}, \oplus)}, w \models \varphi_{i}$ if and only if $\operatorname{apath}_{G}(w, T)$.

The proof proceeds by induction on $i$. The base case $i=m$ is straightforward. For the inductive step $i<m$, consider $w \in V_{i}$.

$K_{(\mathrm{EG}, \oplus)}, w \models \mathrm{EG}\left(s_{i} \oplus s_{i+2} \oplus \hat{s}_{i} \oplus \hat{s}_{i+1} \oplus \varphi_{i+1}\right)\left(=\varphi_{i}\right)$ if and only if there exists an infinite path $\pi \in \Pi(w)$ such that $K_{(\mathrm{EG}, \oplus)}, \pi[j] \models s_{i} \oplus s_{i+2} \oplus \hat{s}_{i} \oplus \hat{s}_{i+1} \oplus \varphi_{i+1}$ $\left(=: \alpha_{i}\right)$ for all $j$. Notice that this is equivalent to $K_{(\mathrm{EG}, \oplus)}, \pi[j] \models \varphi_{i}$ for all $j$.

Assume that such a $\pi$ exists. Since $K_{(\mathrm{EG}, \oplus)}, w \models s_{i}$ and $K_{(\mathrm{EG}, \oplus)}, w \not \models$ $s_{i+2}, \hat{s}_{i}, \hat{s}_{i+1}, \varphi_{i+1}$, it holds that $\pi[1] \models \alpha_{i}$. For the "right neighbour" $v \in \hat{V}_{i}$ of $w$ holds $K_{(\mathrm{EG}, \oplus)}, v \not \forall \varphi_{i}$ (Claim 24(2)). This means that $\pi[2] \in V_{i+1}$. Then $s_{i+1} \in \xi(\pi[2])$ and $s_{i}, s_{i+2}, \hat{s}_{i}, \hat{s}_{i+1} \notin \xi(\pi[2])$. Therefore, $K_{(\mathrm{EG}, \oplus)}, \pi[2] \models \alpha_{i}$ if and only if $K_{(\mathrm{EG}, \oplus)}, \pi[2] \models \varphi_{i+1}$.

Since no node in layer $V_{i+2}^{b}$ satisfies $\varphi_{i}$ (Claim [25), we conclude that $\pi[3]$, $\pi[4], \ldots$ must be in slice $V_{i+1}^{b}$. If $(\pi[3], \pi[3]) \in E^{b}$, we are done as $K_{(\mathrm{EG}, \oplus)}, \pi[3] \models$ $\varphi_{i}$ by Claim 24(33). Otherwise, $\pi[3]$ is a node in $\hat{V}_{i+1}$. By Claim 24(2) we have $K_{(\mathrm{EG}, \oplus)}, \pi[3] \not \models \varphi_{i+1}$. Since $s_{i}, s_{i+2}, \hat{s}_{i} \notin \xi(\pi[3])$ and $\hat{s}_{i+1} \in \xi(\pi[3])$, we get $K_{(\mathrm{EG}, \oplus)}, \pi[3] \models \alpha_{i}$.

Now, $\pi[q]$ for even $q \geq 4$ can be dealt like $\pi[2]$, and $\pi[r]$ for odd $r \geq 5$ can be dealt like $\pi[3]$. Let $\pi[1], \pi[2], \ldots, \pi[5]$ be the finite prefix of $\pi$ that ends in the node through which $\pi$ eventually loops. We have seen that $K_{(\mathrm{EG}, \oplus)}, w \models \varphi_{i}$ if and only if $K_{(\mathrm{EG}, \oplus)}, \pi[2] \models \varphi_{i+1}$ and $K_{(\mathrm{EG}, \oplus)}, \pi[4] \models \varphi_{i+1}$. By the inductive hypothesis this is equivalent to $\operatorname{apath}_{G}(\pi[2], T)$ and apath $_{G}(\pi[4], T)$. Since $\pi[2]$ and $\pi[4]$ are all the successors of $\pi[1]=w$ in $G$, the latter is equivalent to $\operatorname{apath}_{G}(w, T)$.

With Claim 26 we get that $\langle G, s, T\rangle \in \operatorname{ASGAP}\left(\forall_{\text {out }}=2, \exists_{\mathrm{in}}=1\right)$ if and only if $K_{(\mathrm{EG}, \oplus)}, s \models \varphi_{0}$. The CTL-MC(EG, $\left.\oplus\right)$ instance $\left\langle K_{(\mathrm{EG}, \oplus)}, s, \varphi_{0}\right\rangle$ can be computed 
in space logarithmic in the size of $G$. Thus $\operatorname{ASGAP}\left(\forall_{\text {out }}=2, \exists_{\mathrm{in}}=1\right)$ logspace reduces to $\mathrm{CTL}-\mathrm{MC}(\mathrm{EG}, \oplus)$.

\section{Lemma 27. CTL-MC(EG) is NL-hard.}

Proof. We give a logspace reduction from the NL-complete graph accessibility problem. Let $(G, s, t)$ be the given GAP instance with $G=(V, E)$. Let $V^{\prime}=$ $\{(u, i)|u \in V, 1 \leq i \leq| V \mid\}$ be a set consisting of $|V|$ copies of every node in $|V|$, and $E^{\prime}$ be a set of edges on $V^{\prime}$ similar to $E$, such that an edge $(u, v) \in E$ leads to edges from the $i$ th copy of $u$ to the $(i+1)$ st of $v$, plus reflexive edges for all $|V|$ th copies, i.e., $E^{\prime}=\{((u, i),(v, i+1))|(u, v) \in E, 1 \leq i<| V \mid\} \cup$ $\{((u,|V|),(u,|V|)) \mid u \in V\}$. The assignment $\xi$ assigns $a$ to all nodes $(u, i) \in V^{\prime}$ with $i<|V|$ or $u=t$. Let $M=\left(V^{\prime}, E^{\prime}, \xi\right)$ be a Kripke model. It is clear that $G$ has an $s$-t-path if and only if $M,(s, 1) \models \mathrm{EG} a$.

Lemma 28. CTL-MC(EG) and CTL-MC(AF) are in NL.

Proof. First note that EG $\cdots \mathrm{EG} p \equiv \mathrm{EG} p$.

The algorithm for CTL-MC(EG) gets input $\left\langle(W, R, \xi), w_{0}, \mathrm{EG}^{k} p\right\rangle$. If $k=0$ (i.e. the formula to check equals $p$ ), it checks whether $w_{0} \in \xi(p)$ and decides accordingly. If $k>0$, the algorithm must verify whether $(W, R)$ has an infinite paths starting in $w_{0}$ on which $p$ is satisfied in every point. The existence of such a path is equivalent to the existence of two paths $w_{0}=v_{1}, v_{2}, \ldots, v_{m}$ and $v_{m}=u_{1}, u_{2}, \ldots, u_{q}=v_{m}$ for some $m, q \leq|W|$ such that $p$ is satisfied by all $v_{i}$ and $u_{i}$. Both paths together form an ultimately periodic infinite path that is searched for. The algorithm first guesses $v_{m}$ and $q$, and then stepwise guesses the paths and verifies that $p$ is satisfied always. This is clearly an NL-algorithm.

Since $K, w_{0} \models \mathrm{AF} p$ iff $K, w_{0} \not \models \mathrm{EG} \neg p$, the above CTL-MC(EG)-algorithm can be used to decide $\overline{\mathrm{CTL}-\mathrm{MC}(\mathrm{AF})}$. Since NL is closed under complement, CTL-MC(AF) is in NL, too.

Lemma 29. CTL-MC $(\mathrm{EG}, \wedge)$ is in $\mathrm{NL}$.

Proof. We first notice that $\mathrm{EG}(\alpha \wedge \mathrm{EG} \beta) \equiv \mathrm{EG}(\alpha \wedge \beta)$. (1) If $K, w \models \mathrm{EG}(\alpha \wedge \mathrm{EG} \beta)$, then there exists a path starting in $w$ on which everywhere $\alpha \wedge \beta$ is satisfied. (2) If $K, w \models \mathrm{EG}(\alpha \wedge \beta)$, then there exists a path $\pi$ starting in $w$ on which everywhere $\alpha \wedge \beta$ is satisfied. Then $K, \pi[m] \models \mathrm{EG} \beta$ (witnessed by $\pi^{m}$ ) for every $m$. Therefore $K, w \models \mathrm{EG}(\alpha \wedge \mathrm{EG} \beta)$.

Due to $\mathrm{EGEG} \alpha \equiv \mathrm{EG} \alpha$ and the above equivalence, every $\{\mathrm{EG}, \wedge\}$-formula can be transformed to an equivalent formula of the form $\alpha \wedge \bigwedge_{\ell=1,2, \ldots, k}$ EG $\beta_{\ell}$, where $\alpha$ and all $\beta_{\ell}$ are conjunctions of atoms. The satisfaction $K, s \nRightarrow=\mathrm{EG} \beta_{\ell}$ can be checked nondeterministally within logspace by guessing the relevant prefix of a looping infinite path that satisfies $\beta_{\ell}$ in every node. Doing this for all EGsubformulas yields an NL-algorithm for CTL-MC $(E G, \wedge)$.

Lemma 30. CTL-MC(EG, $\vee)$ is in $\mathrm{NL}$. 
Proof. Every $\{\mathrm{EG}, \vee\}$-formula can be transformed into an equivalent formula of the form $\alpha \vee \bigvee_{\ell=1,2, \ldots, k} \mathrm{EG} \beta_{\ell}(*)$, where $\alpha$ is a disjunction of atoms and every $\beta_{\ell}$ is a formula of the form (*) (for $k=0$, such a formula is a disjunction of atoms).

Claim 31. Let $K=(W, R, \xi)$ be a Kripke model, $\alpha$ be a disjunction of atoms, and $\beta_{\ell}$ be formulas of the form $(*)$. Then $K, s \models \mathrm{EG}\left(\alpha \vee \bigvee_{\ell=1,2, \ldots, k} \mathrm{EG} \beta_{\ell}\right)$ if and only if

1. there is a path $v_{1}, \ldots, v_{m}$ through $K$ starting in $s$ and of length $m=|W|+1$ such that $K, v_{i} \models \alpha$ for $i=1,2, \ldots, m$, or

2. there is a path $v_{1}, \ldots, v_{m}$ through $K$ starting in $s$ and of length $1 \leq m \leq$ $|W|+1$ such that $K, v_{i} \models \alpha$ for $i=1,2, \ldots, m-1$ and $K, v_{m} \models \mathrm{EG} \beta_{q}$ for some $q$.

Proof. The implication from left to right is straightforward. Consider the other proof direction. If 1 happens, then $R$ contains an edge from $v_{m}$ to some predecessor on the path. Using this loop we get an infinite path that satisfies $\alpha$ on every of its nodes. If 2 happens, then let $u_{1}, u_{2}, \ldots$ be the infinite path starting with $v_{m}=u_{1}$ such that $K, u_{i} \models \beta_{q}$ for all $i \geq 1$. Then $K, u_{i} \models \mathrm{EG} \beta_{q}$ for all $i \geq 1$. Consequently, on every node of the infinite path $v_{1}(=s), \ldots, v_{m}\left(=u_{1}\right), u_{2}, \ldots$ the formula $\alpha \vee \bigvee_{\ell=1,2, \ldots, k} \mathrm{EG} \beta_{\ell}$ is satisfied. Therefore $K, s \models \mathrm{EG}\left(\alpha \vee \bigvee_{\ell=1,2, \ldots, k} \mathrm{EG} \beta_{\ell}\right)$. $\diamond$

Using this claim, an NL-algorithm can proceed as follows. On input $K, s, \alpha \mathrm{V}$ $\bigvee_{\ell=1,2 \ldots, k} \mathrm{EG} \beta_{\ell}$, it accepts if $K, s \models \alpha$. Otherwise, it guesses an $i$ and goes to check $K, s \models \mathrm{EG} \beta_{i}$ where $\left.\beta_{i}=\alpha^{\prime} \vee \bigvee_{\ell=1,2, \ldots, k} \mathrm{EG} \beta_{\ell}^{\prime}\right)$. For this, it guesses which of the two cases of the Claim has to be fulfilled. Case 1 can be verified straightforwardly. For case 2, it guesses the relevant $m$ and $q$, guesses $v_{i}$ and checks that $K, v_{i} \models \alpha^{\prime}$ for $i=1,2, \ldots, m-1$ and eventually recursively checks whether $K, v_{m} \models \mathrm{EG} \beta_{j}^{\prime}$ for some $j$. Since this is a tail recursion whose depth is bounded by the depth of the input formula, it can be performed nondeterministically within logspace.

Lemma 32. CTL-MC(EG, ᄀ) is in NL.

Proof. The following equivalences hold for EG and its dual AF.

1. EG EG $\alpha \equiv$ EG $\alpha$

2. $\mathrm{AF} \mathrm{AF} \alpha \equiv \mathrm{AF} \alpha$

3. EG AF EG $\alpha \equiv$ AF EG $\alpha$

4. AF EG AF $\alpha \equiv \mathrm{EG}$ AF $\alpha$

Proof of 3 If $K, w \models$ EG AF EG $\alpha$, then clearly $K, w \models$ AF EG $\alpha$. For the other direction, assume $K, w \models \operatorname{AF}$ EG $\alpha$. Take some $\pi \in \Pi(w)$. Then $K, \pi[k] \models \mathrm{EG} \alpha$ for some "smallest" $k$ with $K, \pi[i] \not \models \mathrm{EG} \alpha$ for $i=1,2, \ldots, k-1$. Since $K, \pi[1] \models$ AF EG $\alpha$, it follows that $K, \pi[i] \models$ AF EG $\alpha$ for $i=1,2, \ldots, k-1$. Moreover, let $\rho$ be a path that witnesses $K, \pi[k] \models \mathrm{EG} \alpha$. Then $\rho^{j}$ witnesses 
$K, \rho[j] \models \mathrm{EG} \alpha$ for all $j \geq 1$, and from $K, \rho[j] \models \mathrm{EG} \alpha$ follows $K, \rho[j] \models \mathrm{AF}$ EG $\alpha$. Concluding we have for the infinite path $\lambda=(\pi[1](=w), \pi[2], \ldots, \pi[k-1], \rho[1](=$ $\pi[k]), \rho[2], \ldots)$ that $K, \lambda[i] \models \mathrm{AF} \mathrm{EG} \alpha$ for all $i$, what means that $\lambda$ is a witness for $K, w \models \mathrm{EG}$ AF $\mathrm{EG} \alpha$.

The proof of 4 follows from 3 by the duality of AF and EG.

These equivalences yield that every $\{\mathrm{EG}, \neg$-formula with atom $p$ is equivalent to $\mathrm{EG} \mathrm{AF} p$ or AF EG $p$, or to $\mathrm{EG} p$ or $\mathrm{AF} p$, or to $p$, or to one of these formulas where $p$ is replaced by $\neg p$. For a given $\{\mathrm{EG}, \neg\}$-formula it can be checked in logarithmic space to which of these cases the formula belongs.

We first describe an algorithm for the EG AF $p$ case.

The algorithm gets input $\left\langle(W, R, \xi), w_{0}, \mathrm{EGAF} p\right\rangle$. It must verify whether $(W, R)$ has an infinite paths starting in $w_{0}$ on which $\mathrm{AF} p$ is satisfied in every point. The existence of such a path is equivalent to the existence of two paths $w_{0}=v_{1}, v_{2}, \ldots, v_{m}$ and $v_{m}=u_{1}, u_{2}, \ldots, u_{q}=v_{m}$ for some $m, q \leq|W|$ such that $\mathrm{AF} p$ is satisfied by all $v_{i}$ and $u_{i}$. Both paths together form an ultimately periodic infinite path that is searched for. The algorithm first guesses $v_{m}$ and $q$, and then stepwise guesses the paths and verifies that $\mathrm{AF} p$ is satisfied always. This is done by guessing the next $v_{i}$ (resp. $u_{i}$ ), and then starting the (slightly modified) NL-algorithm for CTL-MC(AF) with input $\left\langle(W, R, \xi), v_{i}, \mathrm{AF} p\right\rangle$. If it reaches an accepting configuration, then the next $v_{i}$ (resp. $u_{i}$ ) is guessed etc.

This also yields an NL-algorithm.

The algorithms for the other cases are constructed in the same way. Since NL is closed under complement, all algorithms are NL-algorithms.

\subsection{EF}

Theorem 33. CTL-MC(EF) and $\mathrm{CTL}-\mathrm{MC}(\mathrm{EF}, \mathrm{V})$ are $\mathrm{NL}$-complete.

Proof. It suffices to show NL-hardness of CTL-MC(EF) and containment in NL of CTL-MC(EF, $\vee)$.

NL-hardness of CTL-MC(EF) follows by a reduction from the directed graph accessability problem as follows. Let $\langle(V, E), s, t\rangle$ be an instance of the graph accessability problem-i.e. we want to decide whether graph $(V, E)$ has an $s$ $t$-path. Let $\hat{E}$ be the reflexive closure of $E$. Then $(V, \hat{E})$ is a total graph, and it has an $s$-t-path if and only if $(V, E)$ has some. Define the assignment $\xi$ as $\xi(t)=\{p\}$ and $\xi(w)=\emptyset$ for $w \neq t$. Then $(V, E)$ has an $s$-t-path if and only if $(V, \hat{E}, \xi), s \models \mathrm{EF} p$.

For CTL-MC(EF, $\vee) \in \mathrm{NL}$, note that $\mathrm{EF}(\alpha \vee \mathrm{EF} \beta) \equiv \mathrm{EF}(\alpha \vee \beta)$ and $\mathrm{EF} \alpha \vee$ $\mathrm{EF} \beta \equiv \mathrm{EF}(\alpha \vee \beta)$. Thus, every $\{\mathrm{EF}, \vee\}$-formula can be transformed into an equivalent formula of the form $\alpha \vee \mathrm{EF} \beta$, where $\alpha$ and $\beta$ are disjunctions of atoms. This transformation can be done in logarithmic space. The NL algorithm on input $\left\langle K, w_{0}, \phi\right\rangle$ verifies whether $K, w_{0} \models \alpha$ or guesses a reachable $v$ and verifies $K, v \models \beta$.

Theorem 34. CTL-MC(EF, $\neg)$ is NL-complete. 
Proof. NL-hardness follows from that of CTL-MC(EF) (Theorem 33).

Every $\{\mathrm{EF}, \neg\}$-formula can be rewritten as a formula with EFs and AGs followed by a literal $p$ or $\neg p$. It is clear that $\operatorname{EFEF} \alpha \equiv \operatorname{EF} \alpha$ and AG AG $\alpha \equiv \operatorname{AG} \alpha$. Thus every such formula can be rewritten as one having a prefix of alternating EFs and AGs. With the equivalences of the following claim we can reduce this prefix to length $\leq 3$.

Claim 35. Let $K$ be a Kripke model, $w$ be a node of $K$, and $\alpha$ be a CTL-formula.

1. $K, w \models \mathrm{EFEF} \alpha$ if and only if $K, w \models \mathrm{EF} \alpha$.

2. $K, w \models \mathrm{AGAG} \alpha$ if and only if $K, w \models \mathrm{AG} \alpha$.

3. $K, w \models$ EFAGEFAG $\alpha$ if and only if $K, w \models \operatorname{EFAG} \alpha$.

(11) and (2) are straightforward. For (11), notice that $K, w \models \alpha$ implies $K, w \models$ EF $\alpha$. For (3), we consider both proof directions separately.

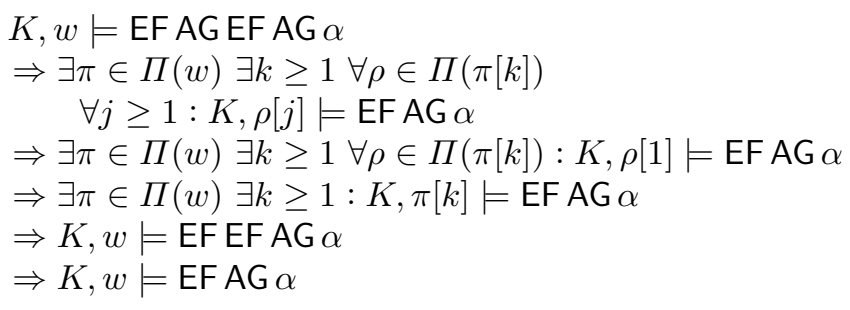

(semantics ...)

(take $j=1$ )

$(\rho[1]=\pi[k])$

(semantics of $\mathrm{EF}$ )

(part (1))

For the other direction, we use (2) and the fact that $K, w \models \beta$ implies $K, w \models \operatorname{EF} \beta$.

$$
\begin{aligned}
& K, w \models \text { EFAG } \alpha \\
& \Rightarrow K, w \models \text { EF AG AG } \alpha \\
& \Rightarrow K, w \models \text { EF AG EF AG } \alpha
\end{aligned}
$$

By Claim 35 follows that every formula in the $\{E F, \neg\}$-fragment has an equivalent formula in the $\{E F, A G\}$-fragment with atomic negation, whith a prefix of at most three temporal operators. Since CTL-MC(EF) and CTL-MC(AG) are in NL (follows from Theorem 33 and the closure of NL under complement), similar as in the proof of Lemma 32, an NL-algorithm can be composed that combines the CTL-MC(EF) and CTL-MC(AG) algorithms in order to evaluate the bounded number of alternations of temporal operators.

Theorem 36. CTL-MC $(\mathrm{EF}, \oplus)$ is $\mathrm{AC}^{1}$-hard.

Proof. We give a reduction from the $\mathrm{AC}^{1}$-complete problem $\mathrm{ASGAP}_{\mathrm{log}}$. Let $\langle G, s, T\rangle$ be an instance of ASGAP ${ }_{\log }$, where $V=V_{\forall} \cup V_{\exists}$ consists of slices $V=V_{0} \cup V_{1} \cup \ldots \cup V_{\ell}$. W.l.o.g. we assume that $V_{\ell} \subseteq V_{\forall}$.

Next we describe the construction of a Kripke model $K_{\mathrm{EF}, \oplus}$ that bases on $G$. In order to ease the readability of the proof, we prefer to use the indices of the slices in reverse order. Let $W_{0}^{\prime}=V_{\ell}$ (the slice with nodes without successors), $W_{1}^{\prime}=V_{\ell-1}, W_{2}^{\prime}=V_{\ell-2}, \ldots, W_{\ell}^{\prime}=V_{0}$. By the above convention, $W_{0}^{\prime}$ consists of $\forall$-nodes. Thus $V_{\forall}=\bigcup_{i \text { even }} W_{i}^{\prime}$ and $V_{\exists}=\bigcup_{i \text { odd }} W_{i}^{\prime}$. Eventually, we add $2(\ell+1)$ 
new nodes and define $W_{i}=W_{i}^{\prime} \cup\left\{a_{i}, b_{i}\right\}$ for $i=0,1, \ldots, \ell$. We will call each $W_{i}$ as layer $i$, and $W=\bigcup_{i=0}^{\ell} W_{i}$ is the set of nodes of $K_{\mathrm{EF}, \oplus \text {. }}$

Next we consider the edges. We take all edges from $E$, and add loops $(u, u)$ for all $u \in W_{0}$. The new $a_{i}$ nodes form a path $\left\{\left(a_{i}, a_{i-1}\right) \mid i=\ell, \ell-1, \ldots, 1\right\}$, and the new $b_{i}$ nodes form a path $E_{b}=\left\{\left(b_{i}, b_{i-1}\right) \mid i=\ell, \ell-1, \ldots, 1\right\}$. Moreover, for odd $i$ every node $u \in W_{i}$ has an edge $\left(u, a_{i-1}\right)$ to $a_{i-1}$, and for even $i \geq 2$ every node $u \in W_{i}$ has an edge $\left(u, b_{i-1}\right)$ to $b_{i-1}$. Let $E^{\prime}$ denote this set of edges.

We complete the description of $K_{\mathrm{EF}, \oplus}$ with the assignment $\xi$. It marks each layer $W_{i}$ with an individual atom $z_{i}$. Moreover, the nodes in $T$ and $b_{0}$ are marked with $t$.

$$
\xi(w)= \begin{cases}\left\{z_{0}, t\right\}, & \text { if } w \in W_{0} \cap\left(T \cup\left\{b_{0}\right\}\right) \\ \left\{z_{i}\right\}, & \text { if } w \in W_{i} \cap \overline{T \cup\left\{b_{0}\right\}}\end{cases}
$$

The Kripke model $K_{\mathrm{EF}, \oplus}$ constructed from $G$ is defined as $K_{\mathrm{EF}, \oplus}=\left(W, E^{\prime}, \xi\right)$. Figure 10 shows an example for the construction. (This example does not have logarithmic depth, but gives a good insight into the construction.)

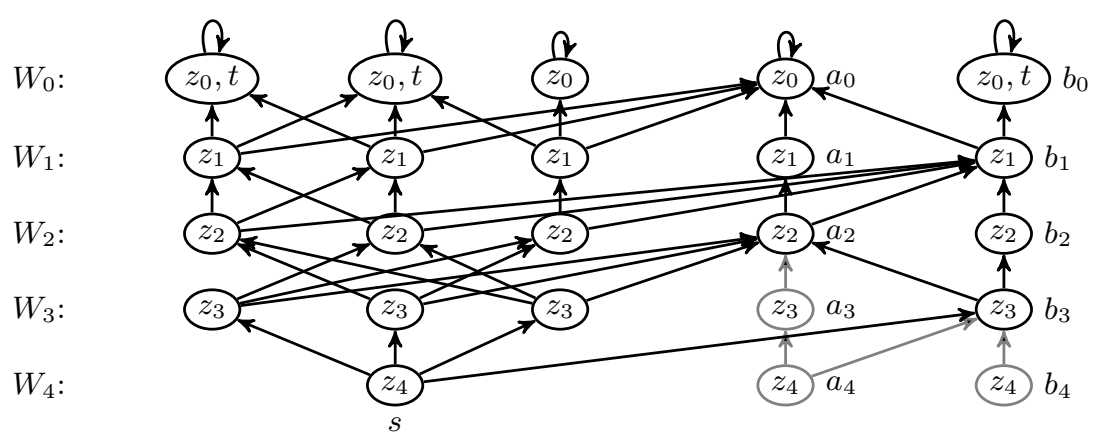

Figure 10. Kripke model $K_{\mathrm{EF}, \oplus}$ constructed from an alternating graph.

For $i=0,1,2, \ldots, \ell$, we inductively define formulas $\varphi_{i}$ as follows. Here we use AG $\alpha$ as abbreviation for $s \oplus \operatorname{EF}(s \oplus \alpha)$ for a new atom $s$ that is satisfied in every node of the Kripke model. Under this condition, $s \oplus \mathrm{EF}(s \oplus \alpha) \equiv \neg \mathrm{EF} \neg \alpha \equiv \mathrm{AG} \alpha$.

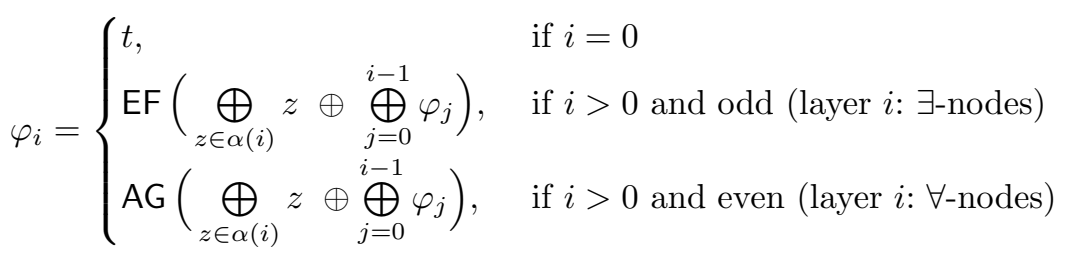

where each $\alpha(i)$ is a subset of $\left\{z_{0}, z_{1}, \ldots, z_{i}\right\}$ defined as follows. Let \#A denote the number of elements of the set $A$. 
- for odd $i$ and $j<i-1$ :

$z_{j} \in \alpha(i) \quad$ iff

$\#\{m \in\{0,1,2, \ldots, j-1\} \mid m$ odd $\}+\#\{m \in\{j+2, \ldots, i-1\} \mid m$ even $\}$ is odd

- for odd $i$ and $j \in\{i-1, i\}$ :

$z_{j} \in \alpha(i) \quad$ iff $\quad \#\{m \in\{0,1,2, \ldots, i-2\} \mid m$ odd $\}$ is odd

- for even $i$ and $j<i-1$ :

$z_{j} \in \alpha(i) \quad$ iff

$\#\{m \in\{0,1,2, \ldots, j-1\} \mid m$ odd $\}+\#\{m \in\{j+2, \ldots, i-1\} \mid m$ even $\}$ is even

- for even $i$ and $j \in\{i-1, i\}$ :

$z_{j} \in \alpha(i) \quad$ iff $\quad \#\{m \in\{0,1,2, \ldots, i-2\} \mid m$ odd $\}$ is odd

As examples, we write down $\varphi_{0}, \varphi_{1}, \varphi_{2}$, and $\varphi_{3}$.

$-\varphi_{0}=t$.

- For $\varphi_{1}: z_{0}, z_{1} \notin \alpha(1)$ since $\#\{m \in \emptyset \mid m$ odd $\}=0$ is even.

Thus, $\varphi_{1}=\mathrm{EF} t$.

- For $\varphi_{2}: z_{0} \in \alpha(2)$ since $\#\{m \in \emptyset \mid m$ odd $\}+\#\{m \in \emptyset \mid m$ even $\}=0$ is even. $z_{1}, z_{2} \notin \alpha(2)$ since $\#\{m \in\{0\} \mid m$ odd $\}=0$ is even.

Thus $\varphi_{2}=\mathrm{AG}\left(z_{0} \oplus t \oplus \mathrm{EF} t\right)$.

- For $\varphi_{3}: z_{0} \in \alpha(3)$ since $\#\{m \in \emptyset \mid m$ odd $\}+\#\{m \in\{2\} \mid m$ even $\}=1$ is odd. $z_{1} \in \alpha(3)$ since $\#\{m \in\{3\} \mid m$ odd $\}+\#\{m \in \emptyset \mid m$ even $\}=1$ is odd. $z_{2}, z_{3} \in \alpha(3)$ since $\#\{m \in\{0,1\} \mid m$ odd $\}=1$ is odd.

Thus $\varphi_{3}=\operatorname{EF}\left(z_{0} \oplus z_{1} \oplus z_{2} \oplus z_{3} \oplus t \oplus \operatorname{EF} t \oplus \operatorname{AG}\left(z_{0} \oplus t \oplus \operatorname{EF} t\right)\right)$.

The following claim contains the crucial properties of Kripke model $K_{\mathrm{EF}, \oplus}$ and the formulas $\varphi_{i}$.

Claim 37. For every $j=0,1, \ldots, \ell$ and every node $w_{j} \in W_{j}$ the following holds.

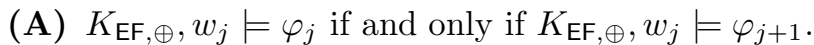

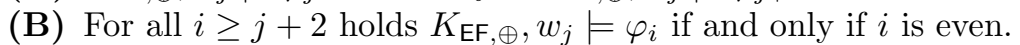

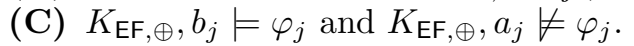

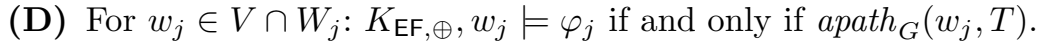

Proof. Throughout the proof, we will use the following straightforward connection between sums of $z_{i}$ and their satisfaction in different layers. By the construc-

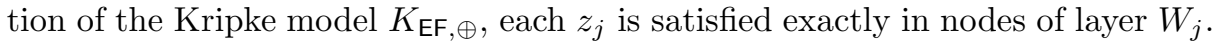
Therefore for all $i \geq j$ and

for every $w_{j} \in W_{j}$ holds: $z_{j} \in \alpha(i)$ if and only if $K_{\mathrm{EF}, \oplus, w_{j}} \models \bigoplus_{z \in \alpha(i)} z$.

The proof of the Claim proceeds by induction on $j$.

The induction base is $j=0$. For case (A), we have to consider $\varphi_{0}=t$ and $\varphi_{1}=\mathrm{EF} t$. In layer $W_{0}$, all nodes only have itself as successor, and therefore $t$ is satisfied in a node of layer $W_{0}$ if and only if $\mathrm{EF} t$ is satisfied by this node. 


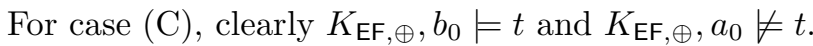

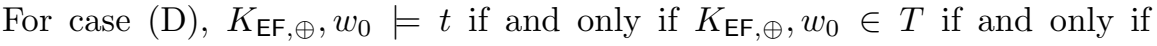
apath $_{G}\left(w_{0}, T\right)$.

For case (B), we proceed by induction on $i$. First, notice that for every $w_{0}$

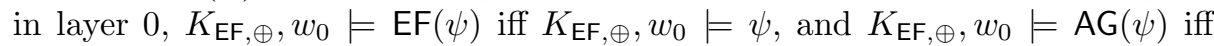
$K_{\mathrm{EF}, \oplus}, w_{0} \models \psi$.

The base case is $i=2$. Every node in layer $W_{0}$ satisfies, $\varphi_{2}=\operatorname{AG}\left(z_{0} \oplus t \oplus \mathrm{EF} t\right)$.

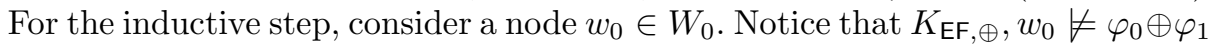
(part (A)). By the inductive hypothesis, all formulas $\varphi_{q}$ for even $q$ with $2 \leq q<i$ are satisfied in $w_{0}$, and all formulas $\varphi_{r}$ for odd $r$ with $2 \leq r<i$ are not satisfied in $w_{0}$. By the semantics of $\oplus$ we can conclude

$$
K_{\mathrm{EF}, \oplus}, w_{0} \models \bigoplus_{l=0}^{i-1} \varphi_{l} \quad \text { if and only if } \quad \#\{m \in\{2,3, \ldots, i-1\} \mid m \text { even }\} \text { is odd. }
$$

We have to consider the cases for odd resp. even $i$ separately, and we start with $i>2$ being odd. By the definition of $\alpha(i)$ we have

$$
z_{0} \in \alpha(i) \text { if and only if } \#\{m \in\{2,3, \ldots, i-1\} \mid m \text { even }\} \text { is odd. }
$$

From (14) and (15) being equivalences with the same right-hand side, and applying (13) for $w_{0}$ in layer $j=0$, we get

$$
K_{\mathrm{EF}, \oplus}, w_{0} \models \bigoplus_{z \in \alpha(i)} z \quad \text { if and only if } \quad K_{\mathrm{EF}, \oplus, w_{0}} \models \bigoplus_{l=0}^{i-1} \varphi_{l},
$$

and therefore

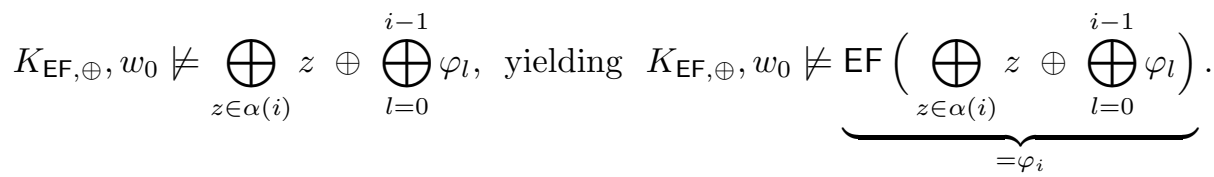

For even $i>2$, we have

$z_{0} \in \alpha(i)$ if and only if $\#\{m \in\{2,3, \ldots, i-1\} \mid m$ even $\}$ is even.

From (14) and (16), and applying (13) for $w_{0}$ in layer $j=0$, we get

$$
K_{\mathrm{EF}, \oplus}, w_{0} \models \bigoplus_{z \in \alpha(i)} z \quad \text { if and only if } \quad K_{\mathrm{EF}, \oplus, w_{0} \forall}^{i-1} \bigoplus_{l=0}^{i} \varphi_{l} .
$$

Therefore,

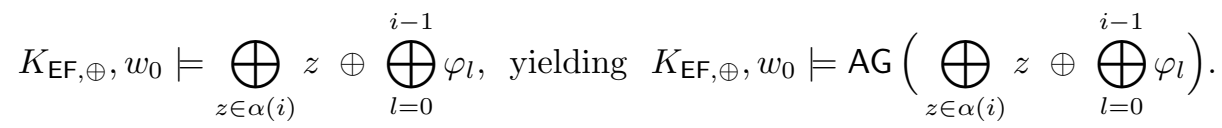


This concludes the proofs of the base cases.

For the induction step, consider $j>0$. We start with some essential observations for nodes $w_{j} \in W_{j}$. For even $i>j$, the formula $\varphi_{i}$ has the form $\operatorname{AG}(\ldots)$,

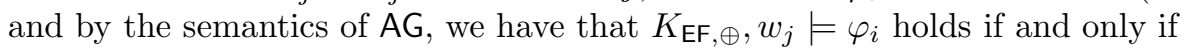

$$
\begin{aligned}
& K_{\mathrm{EF}, \oplus}, w_{j} \models \bigoplus_{z \in \alpha(i)} z \oplus \bigoplus_{l=0}^{i-1} \varphi_{l}, \quad \text { and } \\
& \text { for all successors } v \text { of } w_{j} \text { holds } K_{\mathrm{EF}, \oplus, v \models \varphi_{i}} .
\end{aligned}
$$

Since every successor $v$ of $w_{j}$ is in layer $j-1$, and $i \geq(j-1)+2$, from part (B)

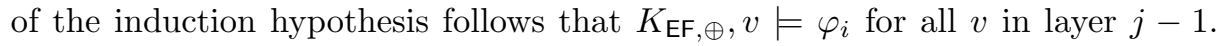
Therefore, $K_{\mathrm{EF}, \oplus}, w_{j} \models \varphi_{i}$ is equivalent to (17). Similarly, for odd $i>j$ holds $K_{\mathrm{EF}, \oplus}, w_{j} \models \varphi_{i}$ if and only if

$$
K_{\mathrm{EF}, \oplus}, w_{j} \models \bigoplus_{z \in \alpha(i)} z \oplus \bigoplus_{l=0}^{i-1} \varphi_{l}, \quad \text { or }
$$

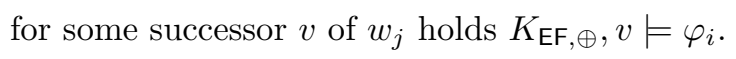

Since every successor $v$ of $w_{j}$ is in layer $j-1$, and $i \geq(j-1)+2$, from part (B)

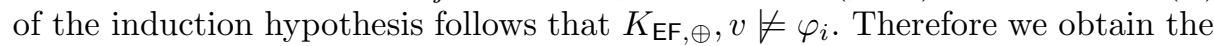
first observation

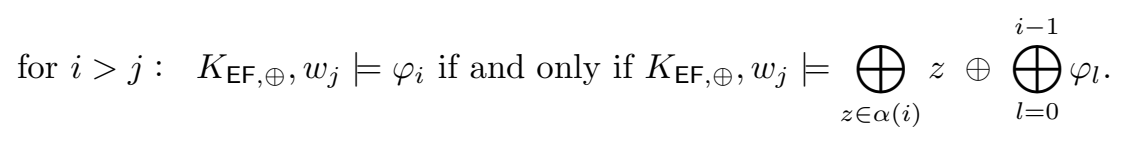

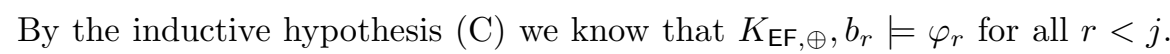
For all odd $r<j$ it holds that $b_{r}$ is reachable from $w_{j}$. Since for odd $r$, the formula $\varphi_{r}$ has the form $\operatorname{EF}(\ldots)$, it follows that $K_{\mathrm{EF}, \oplus}, w_{j} \models \varphi_{r}$ for all odd

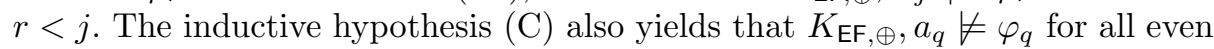
$q<j$. Since all such $a_{q}$ are reachable from $w_{j}$, and for even $q$ the formula $\varphi_{q}$

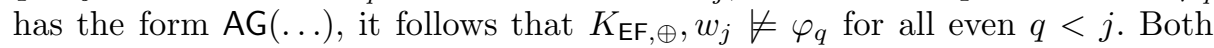
together yield for every $t<j$,

$$
K_{\mathrm{EF}, \oplus}, w_{j} \models \bigoplus_{l=0}^{t} \varphi_{l} \quad \text { if and only if } \quad \#\{m \in\{0,1,2, \ldots, t\} \mid m \text { odd }\} \text { is odd. }
$$

Now back to the inductive step. We start with the inductive step for part (A). Let $w_{j} \in W_{j}$ for $j>0$. By (19) we get

$$
K_{\mathrm{EF}, \oplus}, w_{j} \models \varphi_{j+1} \quad \text { if and only if } \quad K_{\mathrm{EF}, \oplus}, w_{j} \models \bigoplus_{z \in \alpha(j+1)} z \oplus \bigoplus_{l=0}^{j} \varphi_{l} .
$$


Because $z_{j} \in \alpha(j+1)$ if and only if $\#\{m \in\{0,1,2, \ldots, j-1\} \mid m$ odd $\}$ is odd, it follows with (13) and (20) that

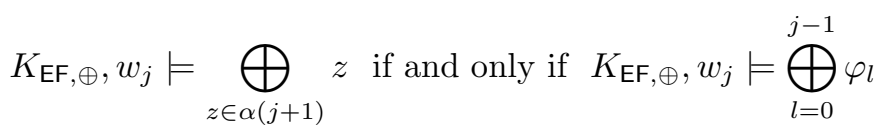

and therefore

$$
K_{\mathrm{EF}, \oplus, w_{j} \not \models} \bigoplus_{z \in \alpha(j+1)} z \oplus \bigoplus_{l=0}^{j-1} \varphi_{l}
$$

Adding $\varphi_{j}$ we get

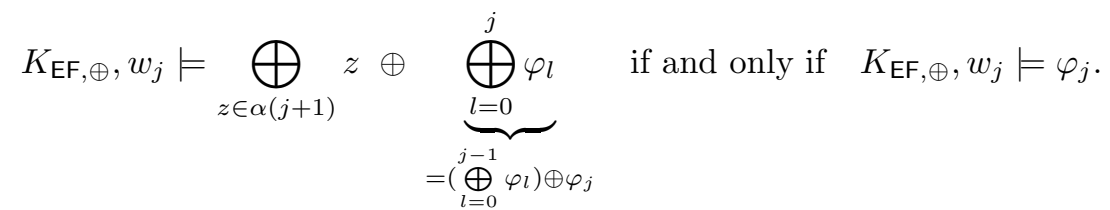

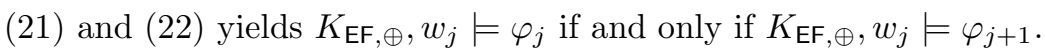

This also proves

$$
\text { for all } j: K_{\mathrm{EF}, \oplus, w_{j} \not \models \varphi_{j} \oplus \varphi_{j+1}} \text {. }
$$

We continue with the induction step for case (B) for $j>0$, and proceed by induction on $i$. The base case is $i=j+2$. By (19) we have

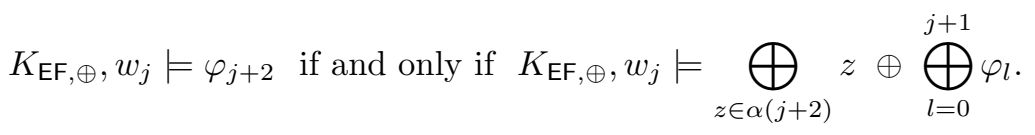

With (23) we get

$$
K_{\mathrm{EF}, \oplus, w_{j}} \models \bigoplus_{z \in \alpha(j+2)} z \oplus \bigoplus_{l=0}^{j+1} \varphi_{l} \text { iff } K_{\mathrm{EF}, \oplus, w_{j}} \models \bigoplus_{z \in \alpha(j+2)} z \oplus \bigoplus_{l=0}^{j-1} \varphi_{l} .
$$

We consider the cases for even resp. odd $j+2$ separately. First, we consider odd $j+2$. Since $\#\{m \in\{j+2, \ldots,(j+2)-1\} \mid m$ even $\}=0$, we get that $z_{j} \in \alpha(j+2)$ iff $\#\{m \in\{0,1,2, \ldots, j-1\} \mid m$ odd $\}$ is odd. With (20) we get

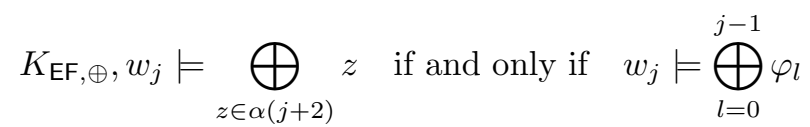

and thus

$$
K_{\mathrm{EF}, \oplus, w_{j} \not \models} \bigoplus_{z \in \alpha(j+2)} z \oplus \bigoplus_{l=0}^{j-1} \varphi_{l}
$$


From (24), (25), and (26) we get $w_{j} \not \models \varphi_{j+2}$ for odd $j$.

For even $j+2$, we proceed similarly. Since \# $\{m \in\{j+2, \ldots,(j+2)-1\} \mid$ $m$ even $\}=0$, we get that $z_{j} \in \alpha(j+2)$ iff $\#\{m \in\{0,1,2, \ldots, j-1\} \mid m$ odd $\}$ is even. With (20) we get

$$
K_{\mathrm{EF}, \oplus, w_{j} \models} \bigoplus_{z \in \alpha(j+2)} z \quad \text { if and only if } \quad K_{\mathrm{EF}, \oplus, w_{j}} \not \bigoplus_{l=0}^{j-1} \varphi_{l}
$$

and thus

$$
K_{\mathrm{EF}, \oplus, w_{j}} \models \bigoplus_{z \in \alpha(j+2)} z \oplus \bigoplus_{l=0}^{j-1} \varphi_{l} .
$$

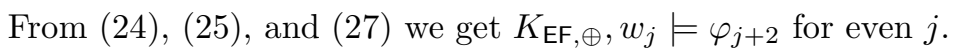

Now for the inductive step $i>j+2$. From (19) we have

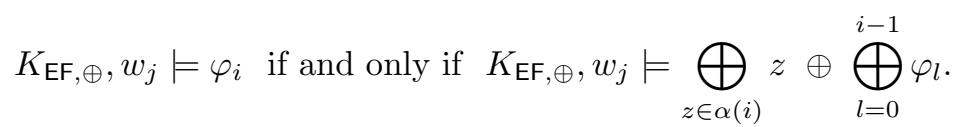

By the inductive hypothesis, we know for $r<i$ holds

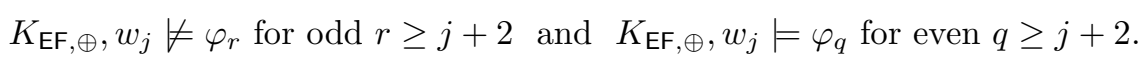

This means

$K_{\mathrm{EF}, \oplus, w_{j}} \models \bigoplus_{l=j+2}^{i-1} \varphi_{l}$ if and only if $\#\{m \in\{j+2, \ldots, i-1\} \mid m$ even $\}$ is odd.

With (20) we get

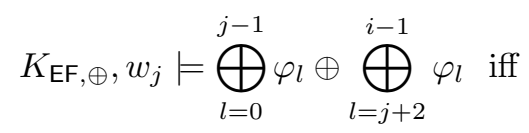

$\#\{m \in\{0,1, \ldots, j-1\} \mid m$ odd $\}+\#\{m \in\{j+2, \ldots, i-1\} \mid m$ even $\}$ is odd.

And with (23) $w_{j} \not \models \varphi_{j} \oplus \varphi_{j+1}$ we eventually get

$$
\begin{aligned}
K_{\mathrm{EF}, \oplus}, w_{j} \models \bigoplus_{l=0}^{i-1} \varphi_{l} \text { iff } & \#\{m \in\{0,1, \ldots, j-1\} \mid m \text { odd }\} \\
& +\#\{m \in\{j+2, \ldots, i-1\} \mid m \text { even }\} \text { is odd. }
\end{aligned}
$$

For odd $i$, we have $z_{j} \in \alpha(i)$ iff $\#\{m \in\{0,1, \ldots, j-1\} \mid m$ odd $\}+\#\{m \in$ $\{j+2, \ldots, i-1\} \mid m$ even $\}$ is odd. With (30) follows

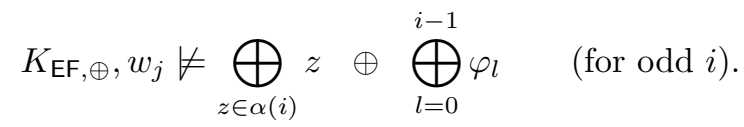




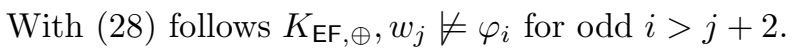

For even $i$, we have $z_{j} \in \alpha(i)$ iff $\#\{m \in\{0,1,2, \ldots, j-1\} \mid m$ odd $\}+\#\{m \in$ $\{j+2, \ldots, i-1\} \mid m$ even $\}$ is even. With (30) follows

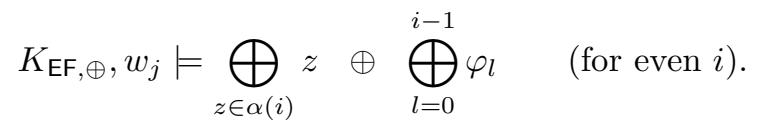

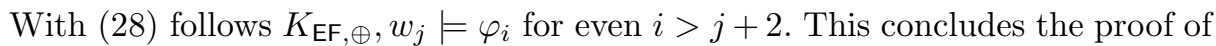
the inductive step for (B).

Now we consider the inductive step for part (C). We start with even $i>0$ and the state $b_{i}$. By the semantics of AG we get

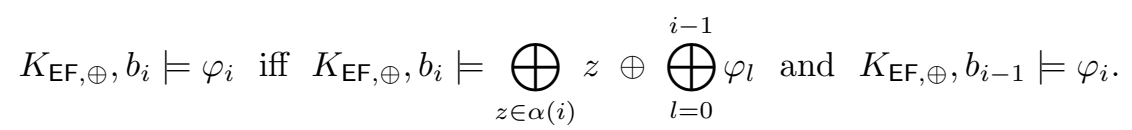

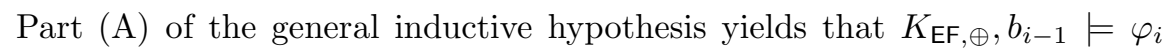

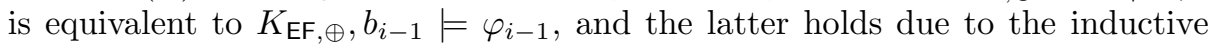
hypothesis. Thus from (31) remains

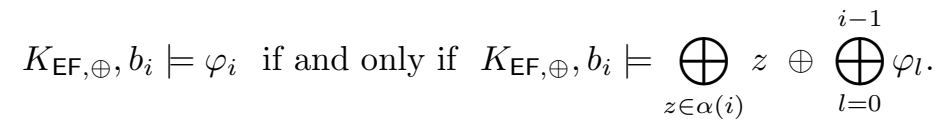

We now consider the right-hand side of (32). With (20) we get

$$
K_{\mathrm{EF}, \oplus}, b_{i} \models \bigoplus_{l=0}^{i-1} \varphi_{l} \text { if and only if } \#\{m \in\{0,1,2, \ldots, i-1\} \mid m \text { odd }\} \text { is odd. }
$$

We have $z_{i} \in \alpha(i)$ iff $\#\{m \in\{0,1,2, \ldots, i-2\} \mid m$ odd $\}$ is odd. Since $i-1$ is odd, we get $z_{i} \in \alpha(i)$ iff $\#\{m \in\{0,1,2, \ldots, i-2, i-1\} \mid m$ odd $\}$ is even. With (33) we get

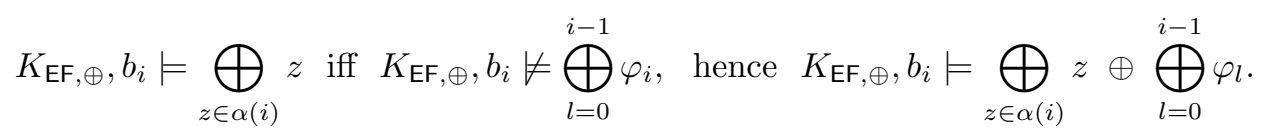

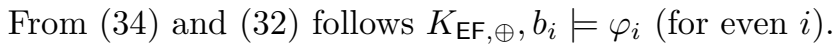

For even $i$ and state $a_{i}$, we have $K_{\mathrm{EF}, \oplus}, a_{i} \models \varphi_{i}$ if and only if

$$
\begin{aligned}
K_{\mathrm{EF}, \oplus}, a_{i} & \models \bigoplus_{z \in \alpha(i)} z \oplus \bigoplus_{l=0}^{i-1} \varphi_{l}, \text { and } \\
K_{\mathrm{EF}, \oplus}, b_{i-1} & \models \varphi_{i}, \quad \text { and } \\
K_{\mathrm{EF}, \oplus, a_{i-1}} & \models \varphi_{i} .
\end{aligned}
$$




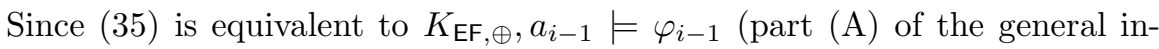

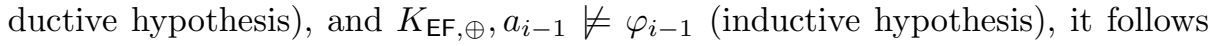
that $K_{\mathrm{EF}, \oplus}, a_{i} \not \models \varphi_{i}$.

Now consider odd $i>0$ and state $b_{i}$. By semantics of $\mathrm{EF}$ we get $K_{\mathrm{EF}, \oplus, b_{i} \models \varphi_{i}}$ if and only if

$$
\begin{aligned}
& K_{\mathrm{EF}, \oplus,}, b_{i} \models \bigoplus_{z \in \alpha(i)} z \oplus \bigoplus_{l=0}^{i-1} \varphi_{l}, \text { or } \\
& K_{\mathrm{EF}, \oplus, b_{i-1}} \models \varphi_{i}, \quad \text { or } \\
& K_{\mathrm{EF}, \oplus, a_{i-1}} \models \varphi_{i} .
\end{aligned}
$$

Part (36) follows from part (A) of the general inductive hypothesis and the inductive hypothesis $K_{\mathrm{EF}, \oplus}, b_{i-1} \models \varphi_{i-1}$. Thus $K_{\mathrm{EF}, \oplus}, b_{i} \models \varphi_{i}$ is proven.

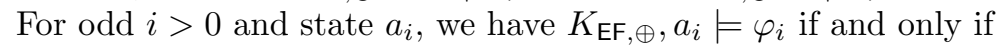

$$
\begin{aligned}
& K_{\mathrm{EF}, \oplus}, a_{i} \models \bigoplus_{z \in \alpha(i)} z \oplus \bigoplus_{l=0}^{i-1} \varphi_{l}, \text { and } \\
& K_{\mathrm{EF}, \oplus, a_{i-1}} \models \varphi_{i} .
\end{aligned}
$$

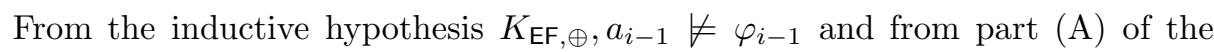

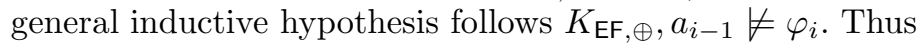

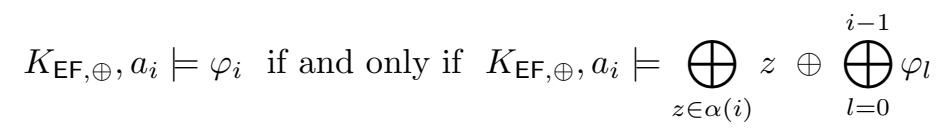

With (20) we have

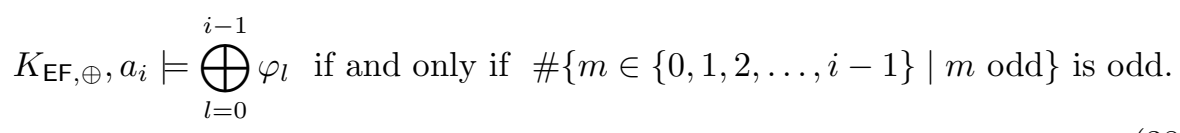

But $z_{i} \in \alpha(i)$ iff $\#\{m \in\{0,1,2, \ldots, i-2\} \mid m$ odd $\}$ is odd. Since $i-1$ is even, we have $z_{i} \in \alpha(i)$ iff $\#\{m \in\{0,1,2, \ldots, i-2, i-1\} \mid m$ odd $\}$ is odd. Thus

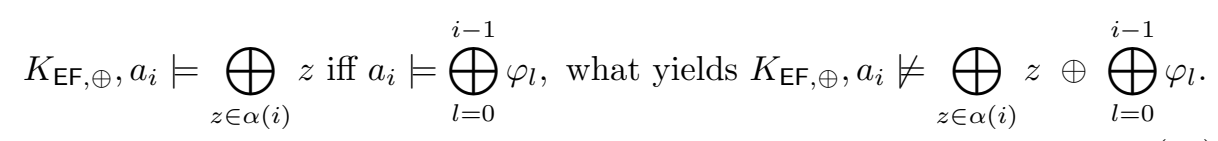

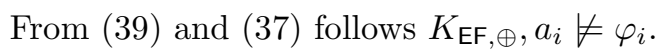

For the inductive step of part (D), let $w_{i} \in W_{i} \cap V$ be a node in layer $i>0$. We start with even $i>0$ and formula $\varphi_{i}$ of the form $\operatorname{AG}(\ldots)$. By the

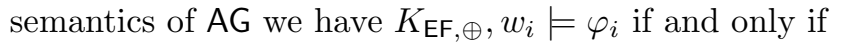

$$
K_{\mathrm{EF}, \oplus}, w_{i} \models \bigoplus_{z \in \alpha(i)} z \oplus \bigoplus_{l=0}^{i-1} \varphi_{l}, \quad \text { and }
$$

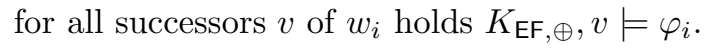


Since all successors $v$ of $w_{i}$ are in layer $i-1$, with the general inductive hypothesis (A) we get that (41) is equivalent to

$$
\text { for all successors } v \text { of } w_{i} \text { holds } K_{\mathrm{EF}, \oplus, v \models \varphi_{i-1}} \text {. }
$$

Since for the successor $b_{i-1}$ of $w_{i}$, the general inductive hypothesis part (C) yields $K_{\mathrm{EF}, \oplus,}, b_{i-1} \models \varphi_{i-1}$, we get that (42) is equivalent to

$$
\text { for all successors } v \in V \cap W_{i-1} \text { of } w_{i} \text { holds } K_{\mathrm{EF}, \oplus}, v \models \varphi_{i-1} \text {. }
$$

Since $z_{i} \in \alpha(i)$ iff $\#\{m \in\{0,1,2, \ldots, i-2\} \mid m$ odd $\}$ is odd, and $i-1$ is odd, we get $z_{i} \in \alpha(i)$ iff $\#\{m \in\{0,1,2, \ldots, i-2, i-1\} \mid m$ odd $\}$ is even. With (20) follows

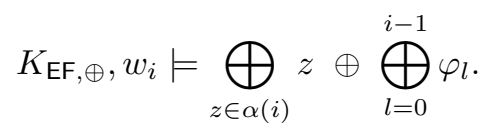

Thus (401) holds, and with the equivalence of (41) and (43) we get

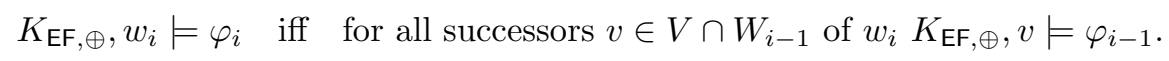

By the inductive hypothesis and the construction of $K_{\mathrm{EF}, \oplus}$ from $G$ we get

$$
K_{\mathrm{EF}, \oplus}, w_{i} \models \varphi_{i} \quad \text { iff } \quad \text { for all successors } v \in V \cap W_{i-1} \text { of } w_{i} \text { in } G \operatorname{apath}_{G}(v, T) .
$$

Since $i$ is even, $w_{i}$ is an $\forall$-node. This yields what we look for, namely

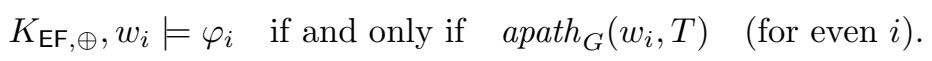

Next we consider odd $i>0$. Then $\varphi_{i}$ has the form $\operatorname{EF}(\ldots)$. From the semantics

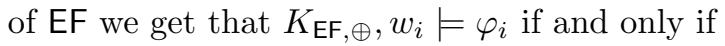

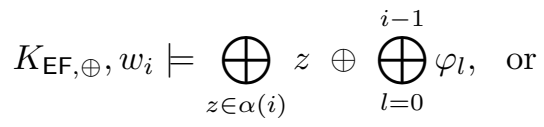

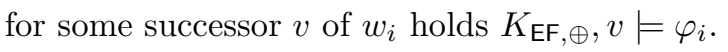

Since $z_{i} \in \alpha(i)$ iff $\#\{m \in\{0,1,2, \ldots, i-2\} \mid m$ odd $\}$ is odd, and $i-1$ is even, we get $z_{i} \in \alpha(i)$ iff $\#\{m \in\{0,1,2, \ldots, i-2, i-1\} \mid m$ odd $\}$ is odd. With (20) follows

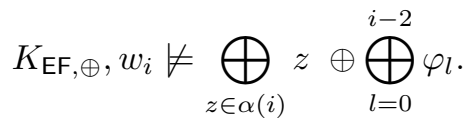

what shows that (44) does not hold. Thus from (44) and (46) we get

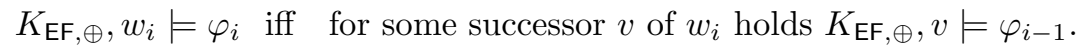


For successor $a_{i-1}$ of $w_{i}$ holds $K_{\mathrm{EF}, \oplus}, a_{i-1} \not \models \varphi_{i}$ (general inductive hypothesis (C), (A)). Therefore

$$
K_{\mathrm{EF}, \oplus}, w_{i} \models \varphi_{i} \text { iff } \quad \text { for some successor } v \in V \cap W_{i-1} \text { of } w_{i} K_{\mathrm{EF}, \oplus, v \models \varphi_{i-1}} \text {. }
$$

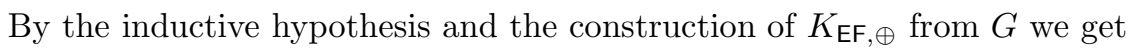

$K_{\mathrm{EF}, \oplus}, w_{i} \models \varphi_{i} \quad$ iff $\quad$ for some successor $v \in V \cap W_{i-1}$ of $w_{i}$ in $G \operatorname{apath}_{G}(v, T)$.

Since $i$ is odd, $w_{i}$ is an $\exists$-node. This concludes the proof of the Claim with

$$
\left.K_{\mathrm{EF}, \oplus}, w_{i} \models \varphi_{i} \quad \text { if and only if } \quad \operatorname{apath}_{G}\left(w_{i}, T\right) \quad \text { (for odd } i\right) .
$$

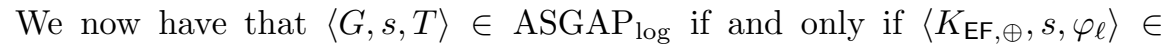
CTL-MC $(E F, \oplus)$. In order to estimate the size $\left|\varphi_{i}\right|$ of formula $\varphi_{i}$, let $\left|\varphi_{i}\right|$ be the number of appearances of atoms in $\varphi$. Then $\left|\varphi_{0}\right|=1$, and $\left|\varphi_{i+1}\right| \leq(i+1)+$ $\sum_{j=0}^{i-1}\left|\varphi_{j}\right|$. This yields $\left|\varphi_{i+1}\right| \leq 2 \cdot\left|\varphi_{i}\right|+1$. Since the depth $\ell$ of $G$ is logarithmic in the size of $G$, we get that $\varphi_{l}$ has size polynomial in the size of $G$. Thus the reduction function described above can be computed in logarithmic space. Since $\mathrm{ASGAP}_{\log }$ is $\mathrm{AC}^{1}$-complete, it follows that CTL-MC(EF, $\left.\oplus\right)$ is $\mathrm{AC}^{1}$-hard under logspace reducibility. 